\title{
Adjoint Higher Order Sensitivities for Fast Full-Wave Optimization of Microwave Filters
}

\author{
Mahmoud A. El Sabbagh, Member, IEEE, Mohamed H. Bakr, Member, IEEE, and John W. Bandler, Fellow, IEEE
}

\begin{abstract}
For the first time, full-wave optimization exploiting adjoint Hessian matrices is applied to the design of microwave filters and transitions. The first- and second-order sensitivities of the scattering parameters are computed analytically using the adjoint network method (ANM). The mode-matching-based ANM is applied to the generalized scattering matrices of the different filter/transition components. Analytical gradient and Hessian matrices of differentiable objective functions are expressed in terms of the first- and second-order response adjoint sensitivities. Optimization techniques exploiting second-order information such as the Levenberg-Marquardt method are applied using the adjoint first- and second-order information. Significant acceleration is achieved using these techniques over gradient-based optimization techniques such as the Broyden-Fletcher-Goldfarb-Shanno method. The adjoint-based sensitivities are also exploited in efficient tolerance analysis of microwave filters.
\end{abstract}

Index Terms-Adjoint networks, computer-aided design (CAD), mode-matching (MM) methods, optimization methods, sensitivity analysis.

\section{INTRODUCTION}

$\mathbf{T}$ HE DESIGN process of microwave filters or transitions involves imposing design specifications on the scattering parameters in certain frequency bands. An optimization algorithm is utilized to obtain a feasible optimal design. This algorithm carries out a number of iterations starting from an initial design. The optimizer invokes the electromagnetic (EM) simulator in each iteration to check the design feasibility and to obtain derivative information necessary to generate the next design. Derivative-based optimization algorithms can exploit first- or second-order derivatives [1]. Algorithms exploiting first-order derivatives when properly modified to exploit available secondorder derivative information are expected to deliver faster convergence. Second-order derivatives, however, are expensive to calculate using central difference (CD) approximations.

The adjoint network method (ANM) is an efficient technique for sensitivity (derivative) estimation [2]-[8]. Using only the original simulation, the first-order sensitivities of the network functions with respect to (w.r.t.) all the design variables are ob-

Manuscript received January 8, 2006; revised April 20, 2006.

M. A. El Sabbagh was with the Department of Electrical and Computer Engineering, McMaster University, Hamilton, ON, Canada L8S 4K1. He is now with the Faculty of Engineering, Department of Electronics and Communication Engineering, Ain Shams University, Abbassia, Cairo 11517, Egypt, and also with the Electrical and Computer Engineering Department, University of Waterloo, Waterloo, ON, Canada N2L 3G1 (e-mail: msabbagh@ieee.org).

M. H. Bakr is with the Department of Electrical and Computer Engineering, McMaster University, Hamilton, ON, Canada L8S 4K1.

J. W. Bandler is with the Department of Electrical and Computer Engineering, McMaster University, Hamilton, ON, Canada L8S 4K1, and also with Bandler Corporation, Dundas, ON, Canada L9H 5E7.

Digital Object Identifier 10.1109/TMTT.2006.877814 tained. These sensitivities give an indication of how critically the designed circuit depends on the design parameters. They are essential in optimization, yield analysis, and tolerance analysis.

Formulations of first- and second-order sensitivities w.r.t. network parameters in terms of wave variables based on the ANM were presented in [5]. Formulations of first- and second-order sensitivities of voltage in terms of circuit parameters were presented in several papers, e.g., [6]-[9]. In [7], third-order sensitivity of voltage in terms of circuit admittance matrix was obtained. ANM-based full-wave optimization of microwave circuits exploiting admittance matrices was introduced in [10]. The design of corrugated feed horn antennas using ANM was discussed in [11]. In [10] and [11], gradient-based optimization techniques were utilized and the derivatives of individual network components were determined by numerical differences.

In this paper, the ANM is applied to calculate first- and second-order sensitivities of the scattering parameters obtained with the full-wave mode-matching (MM) technique. Using only the MM simulation of the original network, first- and second-order sensitivities of the complex scattering parameters and their magnitudes w.r.t. all designable parameters are obtained. Closed-form formulation for the sensitivity analysis of microwave filters and transitions is derived. The formulation exploits generalized scattering matrices (GSMs) of individual components obtained with the full-wave MM technique. GSMs contain entries for both propagating and evanescent modes. Including higher order modes improves the accuracy of both the scattering parameters and their estimated sensitivities. The ANM-based first- and second-order sensitivities are integrated with the Levenberg-Marquardt (LM) optimization method. This method is then exploited in the design of microwave filters. Results show that convergence of the design process using the ANM-based second-order information is significantly faster than gradient-based algorithms such as the quasi-Newton Broyden-Fletcher-Goldfarb-Shanno (BFGS) method.

We start by briefly reviewing the ANM-based approach for estimating first-order sensitivities in Section II. In Section III, it is shown how second-order derivatives are obtained using the ANM. In Section IV, the ANM-based sensitivities are exploited to estimate the first- and second-order sensitivities of differentiable objective functions. The numerical results and discussions are presented in Section V. Finally, conclusions are drawn in Section VI.

\section{ADJOINT FIRST-ORDER DERIVATIVES}

The ANM enables efficient estimation of the sensitivities of the scattering parameters w.r.t. the designable parameters. In 


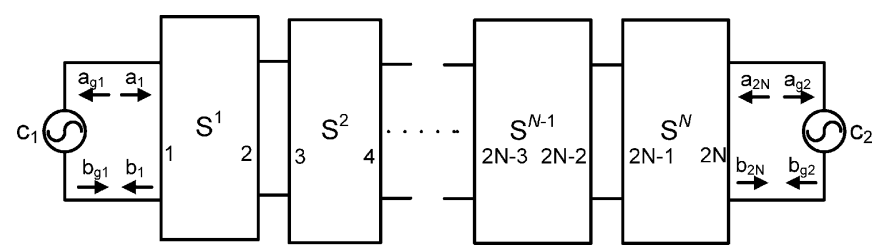

Fig. 1. Network representation of $N$ two-port-components of a microwave circuit.

[5], [12], and [13], it was shown how to get the first-order sensitivity (derivative) of the scattering parameters. In [14], the first-order sensitivity of ridge waveguide scattering parameters w.r.t. the relative dielectric constant was formulated and applied. Here, the ANM is briefly reviewed.

Consider the network representation of a microwave circuit shown in Fig. 1. It is composed of a cascade of $N$ two-port components and independent generators at the input and output. The first-order derivative of the incident wave vector, w.r.t. a design parameter $x$, is [12]

$$
\frac{\partial \boldsymbol{a}}{\partial x}=\mathbf{W}^{-1} \frac{\partial \mathbf{S}^{\text {tot }}}{\partial x} \boldsymbol{a}
$$

where $\boldsymbol{a}$ is the incident wave vector. The connection scattering matrix $\mathbf{W}$ is given by

$$
\mathbf{W}=\mathbf{\Gamma}-\mathbf{S}^{\text {tot }}
$$

where $\boldsymbol{\Gamma}$ is the connection matrix describing the network topology and $\mathbf{S}^{\text {tot }}$ is the block diagonal system scattering matrix. Its submatrices along the diagonal are the GSMs of the various components of the network.

The sensitivity of the ingoing wave variable $a_{r}$ at the $r$ th port of the network is computed by multiplying the left-hand side of (1) by a row vector $\boldsymbol{e}_{r}^{T}$ [12]

$$
\boldsymbol{e}_{r}=\left[\begin{array}{llllclll}
0 & 0 & \cdots & 0 & 1 & 0 & \cdots & 0
\end{array}\right]^{T}
$$

to obtain

$$
\frac{\partial a_{r}}{\partial x}=\boldsymbol{e}_{r}^{T} \frac{\partial \boldsymbol{a}}{\partial x}=\left[\left(\mathbf{W}^{T}\right)^{-1} \boldsymbol{e}_{r}\right]^{T} \frac{\partial \mathbf{S}^{\text {tot }}}{\partial x} \boldsymbol{a}=\boldsymbol{\alpha}^{T} \frac{\partial \mathbf{S}^{\text {tot }}}{\partial x} \boldsymbol{a}
$$

where

$$
\mathbf{W}^{T} \boldsymbol{\alpha}=\boldsymbol{e}_{r}
$$

is the adjoint system and $\boldsymbol{\alpha}$ is the adjoint incident wave vector.

\section{AdJoint SECOND-ORdER Derivatives}

Taking the derivative of (1) w.r.t. another design parameter $y$, the second-order derivative of the incident wave vector is obtained as follows:

$$
\frac{\partial^{2} \boldsymbol{a}}{\partial x \partial y}=\frac{\partial \mathbf{W}^{-1}}{\partial y} \frac{\partial \mathbf{S}^{\mathrm{tot}}}{\partial x} \boldsymbol{a}+\mathbf{W}^{-1} \frac{\partial^{2} \mathbf{S}^{\mathrm{tot}}}{\partial x \partial y} \boldsymbol{a}+\mathbf{W}^{-1} \frac{\partial \mathbf{S}^{\mathrm{tot}}}{\partial x} \frac{\partial \boldsymbol{a}}{\partial y} .
$$

Using the relationship [13]

$$
\frac{\partial \mathbf{W}^{-1}}{\partial y}=-\mathbf{W}^{-1} \frac{\partial \mathbf{W}}{\partial y} \mathbf{W}^{-1}
$$

and with simple manipulations, (6) becomes

$$
\begin{aligned}
\frac{\partial^{2} \boldsymbol{a}}{\partial x \partial y}=\mathbf{W}^{-1}\left[\frac{\partial \mathbf{S}^{\mathrm{tot}}}{\partial y} \mathbf{W}^{-1} \frac{\partial \mathbf{S}^{\mathrm{tot}}}{\partial x}\right. & +\frac{\partial^{2} \mathbf{S}^{\mathrm{tot}}}{\partial x \partial y} \\
& \left.+\frac{\partial \mathbf{S}^{\mathrm{tot}}}{\partial x} \mathbf{W}^{-1} \frac{\partial \mathbf{S}^{\mathrm{tot}}}{\partial y}\right] \boldsymbol{a} .
\end{aligned}
$$

Similar to (4), the second-order derivative of the ingoing wave variable $a_{r}$ at the $r$ th port of the network is

$$
\begin{aligned}
\frac{\partial^{2} a_{r}}{\partial x \partial y}= & \boldsymbol{e}_{r}^{T} \frac{\partial^{2} \boldsymbol{a}}{\partial x \partial y} \\
=\boldsymbol{e}_{r}^{T} \mathbf{W}^{-1}[ & {\left[\frac{\partial \mathbf{S}^{\text {tot }}}{\partial y} \mathbf{W}^{-1} \frac{\partial \mathbf{S}^{\text {tot }}}{\partial x}+\frac{\partial^{2} \mathbf{S}^{\text {tot }}}{\partial x \partial y}\right.} \\
& \left.+\frac{\partial \mathbf{S}^{\mathrm{tot}}}{\partial x} \mathbf{W}^{-1} \frac{\partial \mathbf{S}^{\text {tot }}}{\partial y}\right] \boldsymbol{a} .
\end{aligned}
$$

Equation (9) is reformulated using (1) and (5) as

$$
\frac{\partial^{2} a_{r}}{\partial x \partial y}=\boldsymbol{\alpha}^{T}\left[\frac{\partial \mathbf{S}^{\text {tot }}}{\partial y} \frac{\partial \boldsymbol{a}}{\partial x}+\frac{\partial^{2} \mathbf{S}^{\text {tot }}}{\partial x \partial y} \boldsymbol{a}+\frac{\partial \mathbf{S}^{\text {tot }}}{\partial x} \frac{\partial \boldsymbol{a}}{\partial y}\right] .
$$

The scattering parameter $S_{i j}$ is given by

$$
S_{i j}=\left.b_{i}\right|_{a_{j}=1}=a_{r} .
$$

In (11), $a_{r}$ is the ingoing wave variable at the $r$ th port with a matched load terminating the $i$ th port of the network. The condition $a_{j}=1$ is imposed by the matched generator with $c=1$ connected to port $j$ (see Fig. 1). The adjoint network is excited at port $r$ by a matched generator with impressed wave $\alpha_{i}=1$ [12]. Thus,

$$
\begin{aligned}
\frac{\partial S_{i j}}{\partial x} & =\frac{\partial a_{r}}{\partial x} \\
\frac{\partial^{2} S_{i j}}{\partial x \partial y} & =\frac{\partial^{2} a_{r}}{\partial x \partial y} .
\end{aligned}
$$

The derivatives of the system scattering matrix $\mathbf{S}^{\text {tot }}$ w.r.t. design parameters $x$ and/or $y$ are obtained analytically. For example, consider one of its blocks to be the GSM of a transmission line of length $L_{i},(i=1,2, \ldots)$ with $M$ modes in each port

$$
\mathbf{S}^{L_{i}}=\left[\begin{array}{cc}
\mathbf{0} & \boldsymbol{\Gamma}_{\gamma} \\
\boldsymbol{\Gamma}_{\gamma} & \mathbf{0}
\end{array}\right]
$$

where $\mathbf{S}^{L_{i}} \in \mathbb{C}^{2 M \times 2 M}$, and $\boldsymbol{\Gamma}_{\gamma} \in \mathbb{C}^{M \times M}$ is a diagonal matrix given by

$$
\boldsymbol{\Gamma}_{\gamma}=\left[\begin{array}{cccc}
e^{-\gamma_{1} L_{i}} & 0 & \cdots & 0 \\
0 & e^{-\gamma_{2} L_{i}} & \cdots & 0 \\
\vdots & \vdots & \vdots & \vdots \\
0 & 0 & \cdots & e^{-\gamma_{M} L_{i}}
\end{array}\right]
$$

and $\gamma_{j}(j=1,2, \ldots, M)$ is the propagation constant of the $j$ th mode in each port. Here, it is assumed that all the connected 
ports have the same number of modes. The first-order derivative of the GSMs $\mathbf{S}^{L_{i}}$ w.r.t. the length $L_{i}$ is given by

$$
\frac{\partial}{\partial L_{i}} \mathbf{S}^{L_{i}}=\left[\begin{array}{cc}
\mathbf{0} & -\boldsymbol{\gamma} \boldsymbol{\Gamma}_{\gamma} \\
-\boldsymbol{\gamma} \boldsymbol{\Gamma}_{\gamma} & \mathbf{0}
\end{array}\right]
$$

where

$$
\boldsymbol{\gamma}=\left[\begin{array}{cccc}
\gamma_{1} & 0 & \cdots & 0 \\
0 & \gamma_{2} & \cdots & 0 \\
\vdots & \vdots & \vdots & \\
0 & 0 & \cdots & \gamma_{M}
\end{array}\right]
$$

The second-order derivative of the GSM $\mathbf{S}^{L_{i}}$ w.r.t. the length $L_{i}$ is given by

$$
\frac{\partial^{2} \mathbf{S}^{L_{i}}}{\partial L_{i}^{2}}=\left[\begin{array}{cc}
\mathbf{0} & \boldsymbol{\gamma}^{2} \boldsymbol{\Gamma}_{\gamma} \\
\boldsymbol{\gamma}^{2} \boldsymbol{\Gamma}_{\gamma} & \mathbf{0}
\end{array}\right]
$$

\section{OPTIMIZATION}

As described in Sections II and III, the ANM is used to obtain the first- and second-order derivatives of the scattering parameters. The Jacobian and Hessian matrices of differentiable objective functions are expressed in terms of these derivatives. Here, how the analytical Hessian matrices are obtained and implemented in optimization is presented. The error function to be minimized is defined as a weighted $L_{2}$ norm of the difference between the actual and desired response of the circuit (sum of squares of nonlinear functions [16]-[18]). The following objective function was successfully used in filter optimization [19]-[21]:

$$
\begin{aligned}
f(\boldsymbol{x}) & =\sum_{l=1}^{N_{\mathrm{pb}}}\left(r_{11}^{(l)}\left(\boldsymbol{x}, f_{\mathrm{pbl} l}\right)\right)^{2}+\sum_{k=1}^{M_{\mathrm{sb}}}\left(r_{21}^{(k)}\left(\boldsymbol{x}, f_{\mathrm{sb} k}\right)\right)^{2} \\
& =\boldsymbol{r}_{11}^{T} \boldsymbol{r}_{11}+\boldsymbol{r}_{21}^{T} \boldsymbol{r}_{21}=\boldsymbol{r}^{T} \boldsymbol{r},
\end{aligned}
$$

where $f_{\mathrm{pb} l}$ is the $l$ th frequency point in the passband and $f_{\mathrm{sb} k}$ is the $k$ th frequency point in the stopband. $N_{\mathrm{pb}}$ and $M_{\mathrm{sb}}$ are the number of frequency points in the passband and stopband, respectively. $\boldsymbol{r}_{11}$ and $\boldsymbol{r}_{21}$ are the residual vectors corresponding to $\left|S_{11}\right|$ and $\left|S_{21}\right|$, respectively. They are expressed as

$$
\begin{aligned}
\boldsymbol{r}_{11} & =\left[\begin{array}{llll}
r_{11}^{(1)} & r_{11}^{(2)} & \cdots & r_{11}^{\left(N_{\mathrm{pb}}\right)}
\end{array}\right]^{T} \\
\boldsymbol{r}_{21} & =\left[\begin{array}{llll}
r_{21}^{(1)} & r_{21}^{(2)} & \cdots & r_{21}^{\left(M_{\mathrm{sb}}\right)}
\end{array}\right]^{T} \\
\boldsymbol{r} & =\left[\begin{array}{ll}
\boldsymbol{r}_{11}^{T} & \boldsymbol{r}_{21}^{T}
\end{array}\right]^{T} .
\end{aligned}
$$

Here, $\boldsymbol{r} \in \mathbb{R}^{m \times 1}$ and $m=N_{\mathrm{pb}}+M_{\mathrm{sb}}$. The elements of the vectors $\boldsymbol{r}_{11}$ and $\boldsymbol{r}_{21}$ are given by

$$
r_{11}^{(l)}=\left\{\begin{array}{l}
\frac{1}{\sqrt{2}} w_{\mathrm{pb}}\left(\left|S_{11}\left(\boldsymbol{x}, f_{\mathrm{pb} l}\right)\right|-\left|S_{11}^{\mathrm{des}}\right|\right), \\
0, \quad \text { otherwise }
\end{array}\right.
$$

$$
r_{21}^{(k)}=\left\{\begin{array}{l}
\frac{1}{\sqrt{2}} w_{\mathrm{sb}}\left(\left|S_{21}\left(\boldsymbol{x}, f_{\mathrm{sb} k}\right)\right|-\left|S_{21}^{\mathrm{des}}\right|\right), \\
0, \quad \text { otherwise }
\end{array}\right.
$$

where $w_{\mathrm{pb}}$ is the passband weighting factor and $w_{\mathrm{sb}}$ is the stopband weighting factor. $\left|S_{11}^{\text {des }}\right|$ is the absolute value of the desired return loss in the passband. $\left|S_{21}^{\text {des }}\right|$ is the absolute value of the desired attenuation in the stopband. The switching conditions in (20) and (21) are

$$
\begin{aligned}
& \text { cond } 1 \equiv\left|S_{11}\left(\boldsymbol{x}, f_{\mathrm{pbl}}\right)\right| \geq\left|S_{11}^{\text {des }}\right| \\
& \text { cond } 2 \equiv\left|S_{21}\left(\boldsymbol{x}, f_{\mathrm{sb} k}\right)\right| \geq\left|S_{21}^{\text {des }}\right| .
\end{aligned}
$$

The error function $f(\boldsymbol{x})$ is minimized subject to simple lower and upper bounds on the design variables

$$
\begin{aligned}
& \min _{\boldsymbol{x} \in \mathbb{R}^{n}} f(\boldsymbol{x}) \\
& \text { subject to } \boldsymbol{l} \leq \boldsymbol{x} \leq \boldsymbol{u} .
\end{aligned}
$$

This error function has the gradient

$$
\begin{aligned}
\nabla f(\boldsymbol{x})= & \sum_{l=1}^{N_{\mathrm{pb}}} \sqrt{2} w_{\mathrm{pb}} r_{11}^{(l)}\left(\boldsymbol{x}, f_{\mathrm{pb} l}\right) \frac{\partial\left|S_{11}\left(\boldsymbol{x}, f_{\mathrm{pb} l}\right)\right|}{\partial \boldsymbol{x}} \\
& +\sum_{k=1}^{M_{\mathrm{sb}}} \sqrt{2} w_{\mathrm{sb}} r_{21}^{(k)}\left(\boldsymbol{x}, f_{\mathrm{sb} k}\right) \frac{\partial \mid S_{21}\left(\boldsymbol{x}, f_{\mathrm{sb} k} \mid\right.}{\partial \boldsymbol{x}} \\
= & \mathbf{J}^{T} \boldsymbol{r}
\end{aligned}
$$

and the Hessian matrix

$$
\begin{aligned}
& \nabla^{2} f(x) \\
& =\sum_{l=1}^{N_{\mathrm{pb}}} w_{\mathrm{pb}}^{2}\left[\frac{\partial\left|S_{11}\left(\boldsymbol{x}, f_{\mathrm{pb} l}\right)\right|}{\partial \boldsymbol{x}} \frac{\partial\left|S_{11}\left(\boldsymbol{x}, f_{\mathrm{pb} l}\right)\right|^{T}}{\partial \boldsymbol{x}}\right. \\
& \left.+\left(\left|S_{11}\left(\boldsymbol{x}, f_{\mathrm{pb} l}\right)\right|-\left|S_{11}^{\text {des }}\right|\right) \frac{\partial^{2}\left|S_{11}\left(\boldsymbol{x}, f_{\mathrm{pb} l}\right)\right|}{\partial \boldsymbol{x}^{2}}\right] \\
& +\sum_{k=1}^{M_{\mathrm{sb}}} w_{\mathrm{sb}}^{2}\left[\frac{\partial\left|S_{21}\left(\boldsymbol{x}, f_{\mathrm{sb} k}\right)\right|}{\partial \boldsymbol{x}} \frac{\partial\left|S_{21}\left(\boldsymbol{x}, f_{\mathrm{sb} k}\right)\right|^{T}}{\partial \boldsymbol{x}}\right. \\
& \left.+\left(\left|S_{21}\left(\boldsymbol{x}, f_{\mathrm{sb} k}\right)\right|-\left|S_{21}^{\text {des }}\right|\right) \frac{\partial^{2}\left|S_{21}\left(\boldsymbol{x}, f_{\mathrm{sb} k}\right)\right|}{\partial \boldsymbol{x}^{2}}\right] \\
& =\mathbf{J}^{T} \mathbf{J}+\sum_{k=1}^{m} r_{k}(\boldsymbol{x}) \nabla^{2} r_{k}(\boldsymbol{x})
\end{aligned}
$$

where $\mathbf{J} \in \mathbb{R}^{m \times n}$ is the Jacobian of $\boldsymbol{r}$ defined by

$$
J_{k l}=\frac{\partial r_{k}}{\partial x_{l}} ; \quad 1 \leq k \leq m ; \quad 1 \leq l \leq n .
$$

The expressions of the gradient in (23) and the Hessian in (24) require the first- and second-order derivatives of the magnitude of the scattering parameters. The first-order derivative of the magnitude, w.r.t. a variable $x$, is given as [13]

$$
\frac{\partial\left|S_{i j}\right|}{\partial x}=\operatorname{Re}\left(\frac{\left|S_{i j}\right|}{S_{i j}} \frac{\partial S_{i j}}{\partial x}\right)
$$




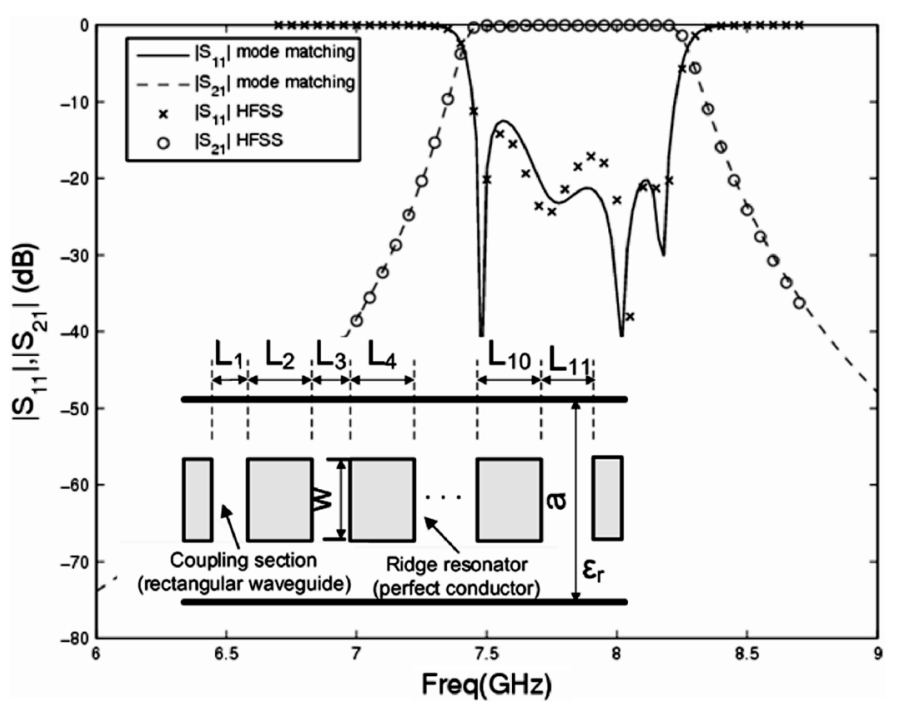

Fig. 2. Top view of the five-pole ridge waveguide filter and its initial frequency response. MM response compared to HFSS [25] response. Waveguide cross section: 0.24 in $\times 0.071$ in, ridge width $w=0.08$ in, ridge gap $=0.0138 \mathrm{in}, \varepsilon_{r}=$ $5.9, L_{1}$ to $L_{11}$ dimensions are as indicated in Table II.

TABLE I

OPTIMIZATION GOALS

\begin{tabular}{|l|c|}
\hline Specification & Value \\
\hline 3-dB passband & $7.26-8.06 \mathrm{GHz}$ \\
passband return loss & 0.005 \\
insertion loss in the band $6.9-7.0 \mathrm{GHz}$ & 0.01 \\
insertion loss in the band $8.4-8.5 \mathrm{GHz}$ & 0.01 \\
\# frequency points in the passband $(N \mathrm{pb})$ & 20 \\
\# frequency points in the band $6.9-7.0 \mathrm{GHz}$ & 5 \\
\# frequency points in the band $8.4-8.5 \mathrm{GHz}$ & 5 \\
Passband weight $\left(w_{\mathrm{pb}}\right)$ & 2 \\
Stopband weight $\left(w_{\mathrm{sb}}\right)$ & 1 \\
\hline
\end{tabular}

TABLE II

DIMENSIONS OF $\boldsymbol{x}$ (IN INCHES) BEFORE AND AFTER OPTIMIZATION $L_{7}=L_{5}, L_{8}=L_{4}, L_{9}=L_{3}, L_{10}=L_{2}, L_{11}=L_{1}$

\begin{tabular}{|l|c|c|c|c|c|c|}
\hline & $L_{1}$ & $L_{2}$ & $L_{3}$ & $L_{4}$ & $L_{5}$ & $L_{6}$ \\
\hline Initial & 0.07 & 0.05 & 0.21 & 0.03 & 0.25 & 0.03 \\
\hline BFGS & 0.0617 & 0.0623 & 0.1859 & 0.0383 & 0.2383 & 0.0366 \\
\hline LM & 0.0712 & 0.0563 & 0.2022 & 0.0368 & 0.2458 & 0.0357 \\
\hline
\end{tabular}

Taking the derivative of (26) w.r.t. $y$ and after simple algebraic manipulations, the second-order derivative of the magnitude is given as (details of the derivation are shown in the Appendix)

$$
\begin{array}{r}
\frac{\partial^{2}\left|S_{i j}\right|}{\partial x \partial y}=\operatorname{Re}\left(\frac{\left|S_{i j}\right|}{S_{i j}} \frac{\partial^{2} S_{i j}}{\partial x \partial y}\right)+\frac{1}{\left|S_{i j}\right|}\left|\frac{\partial S_{i j}}{\partial y}\right|^{2} \operatorname{Re}\left(\frac{\frac{\partial S_{i j}}{\partial x}}{\frac{\partial S_{i j}}{\partial y}}\right) \\
-\frac{1}{\left|S_{i j}\right|} \frac{\partial\left|S_{i j}\right|}{\partial x} \frac{\partial\left|S_{i j}\right|}{\partial y} .
\end{array}
$$

The first-order derivatives that form the Jacobian matrix $\boldsymbol{J}$ are computed using (26). The Jacobian matrix is used to calculate the error function gradient in (23). A distinctive feature of the least squares formulation is that by knowing the Jacobian, the first part of the Hessian $\nabla^{2} f(x)$ is computed for free.

Many optimization techniques such as Gauss-Newton, Newton-Raphson, quasi-Newton, gradient methods, and LM method can be applied to solve the minimization problem in

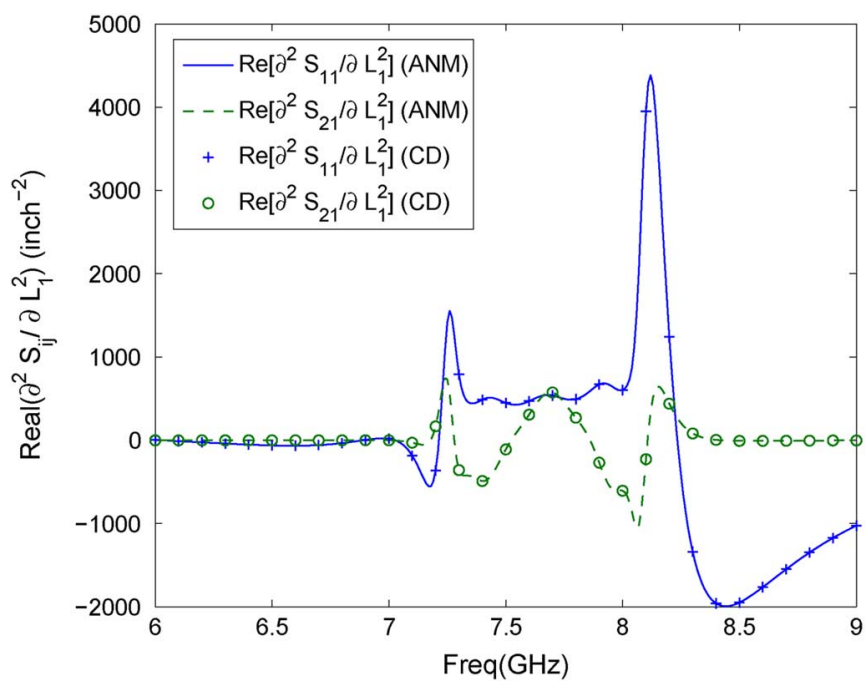

Fig. 3. Real part of the dominant mode scattering parameters secondorder derivatives w.r.t. $L_{1}$ of the five-pole filter with center frequency $f_{0}=7.66 \mathrm{GHz}$, obtained using the ANM and finite CDs. (Color version available online at: http://ieeexplore.ieee.org.)

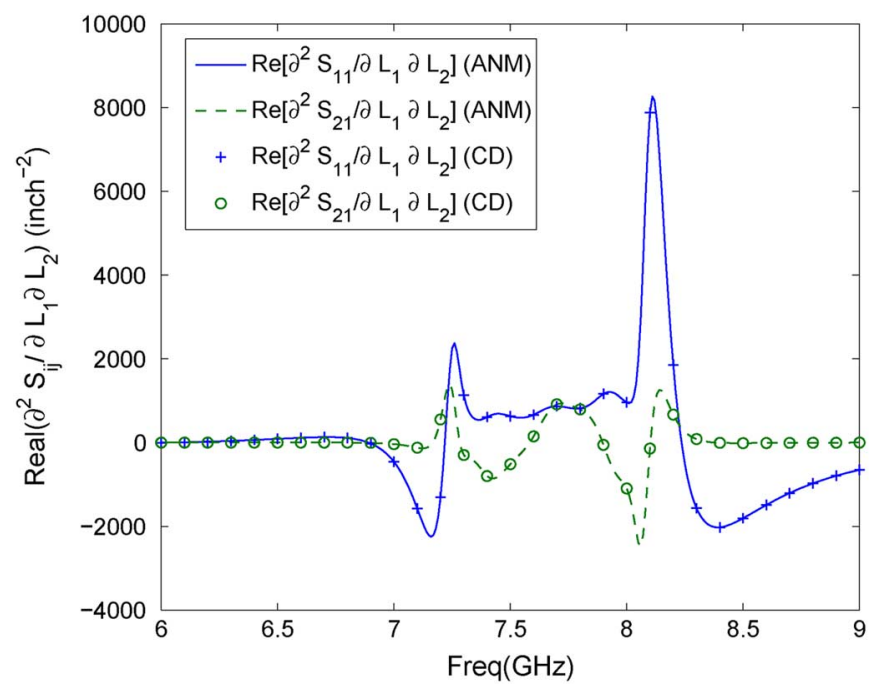

Fig. 4. Real part of the dominant mode scattering parameters second-order derivatives w.r.t. $L_{1}$ and $L_{2}$ of the five-pole filter with center frequency $f_{0}=$ $7.66 \mathrm{GHz}$, obtained using the ANM and finite CDs. (Color version available online at: http://ieeexplore.ieee.org.)

(22). In these techniques, the methods of LM and quasi-Newton prove to be the best ones in most cases in terms of convergence properties. The BFGS method, regarded as the most efficient algorithm among various quasi-Newton methods, is more often adopted to solve unconstrained optimization problems. It presents good performance and does not require the Hessian matrix. However, the line search in the BFGS when applied to complex systems may be time consuming.

Two optimization procedures are compared to each other to show the advantage of having analytical Hessian matrices. These optimization procedures are briefly described below.

\section{A. $B F G S$}

BFGS is a quasi-Newton method used to solve nonlinear problems with simple bounds. It is based on the gradient 


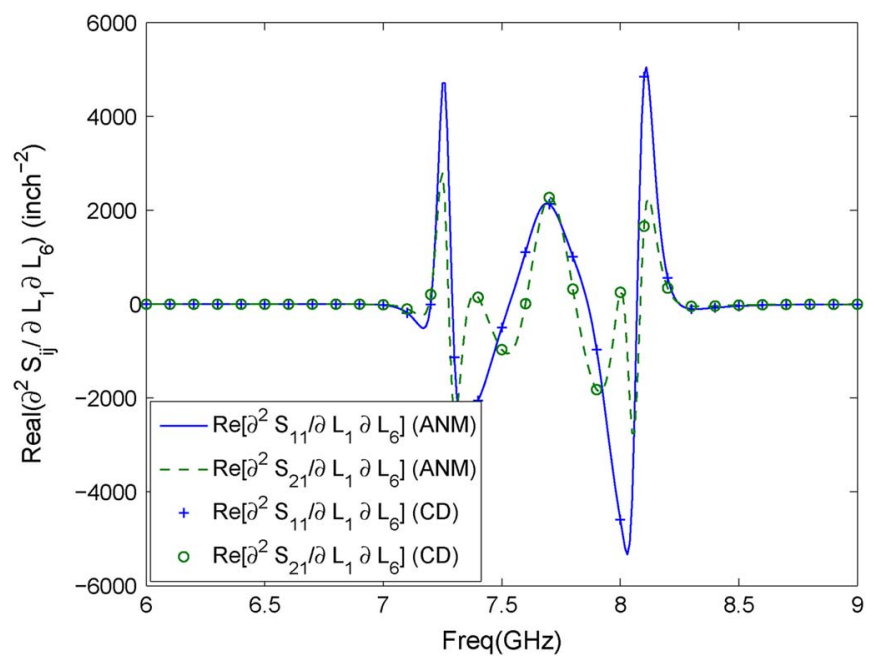

Fig. 5. Real part of the dominant mode scattering parameters second-order derivatives w.r.t. $L_{1}$ and $L_{6}$ of the five-pole filter with center frequency $f_{0}=$ $7.66 \mathrm{GHz}$, obtained using the ANM and finite CDs. (Color version available online at: http://ieeexplore.ieee.org.)

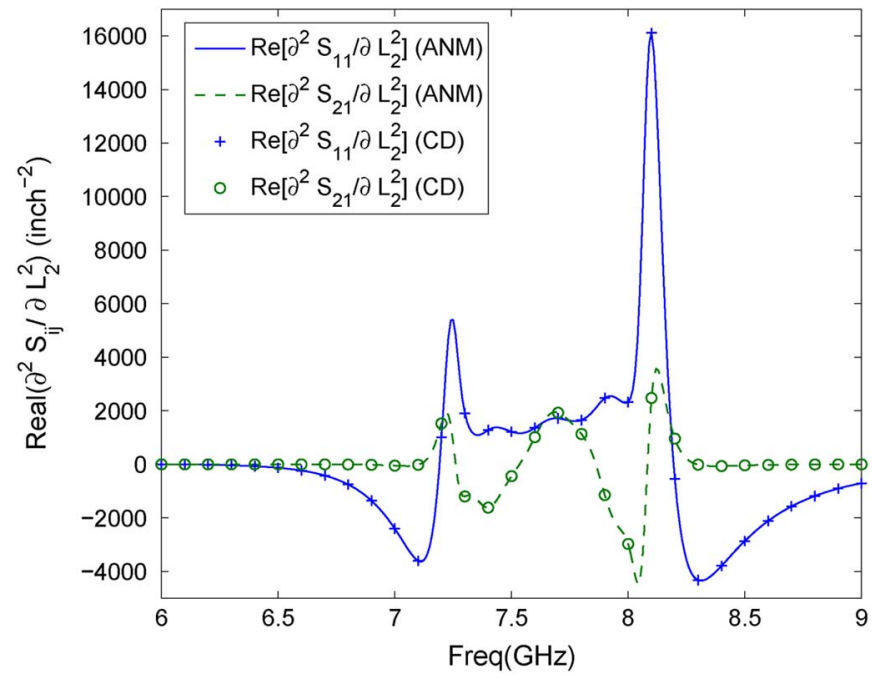

Fig. 6. Real part of the dominant mode scattering parameters secondorder derivatives w.r.t. $L_{2}$ of the five-pole filter with center frequency $f_{0}=7.66 \mathrm{GHz}$, obtained using the ANM and finite CDs. (Color version available online at: http://ieeexplore.iee.org.)

projection method and uses the BFGS update formula to approximate the Hessian matrix of the objective function. The BFGS formula is given as [16]

$$
\mathbf{B}_{k+1}=\mathbf{B}_{k}+\frac{\boldsymbol{y}_{k} \boldsymbol{y}_{k}^{T}}{\boldsymbol{y}_{k}^{T} \boldsymbol{h}_{k}}-\frac{\mathbf{B}_{k} \boldsymbol{h}_{k} \boldsymbol{h}_{k}^{T} \mathbf{B}_{k}}{\boldsymbol{h}_{k}^{T} \mathbf{B}_{k} \boldsymbol{h}_{k}}
$$

where $\boldsymbol{h}_{k}=\boldsymbol{x}_{k+1}-\boldsymbol{x}_{k}$ is the current optimization step and $\boldsymbol{y}_{k}=\boldsymbol{r}\left(\boldsymbol{x}_{k+1}\right)-\boldsymbol{r}\left(\boldsymbol{x}_{k}\right)$. More details about the algorithm can be found in [22] and [23].

\section{B. LM Method}

In this method, the user provides an initial approximation of the vector of design parameters $\boldsymbol{x}=\boldsymbol{x}_{0}$ to the solution of the problem. Usually, the initial solution is obtained from synthesis. The algorithm then determines a correction $h_{k}$ to $x_{k}$ that pro-

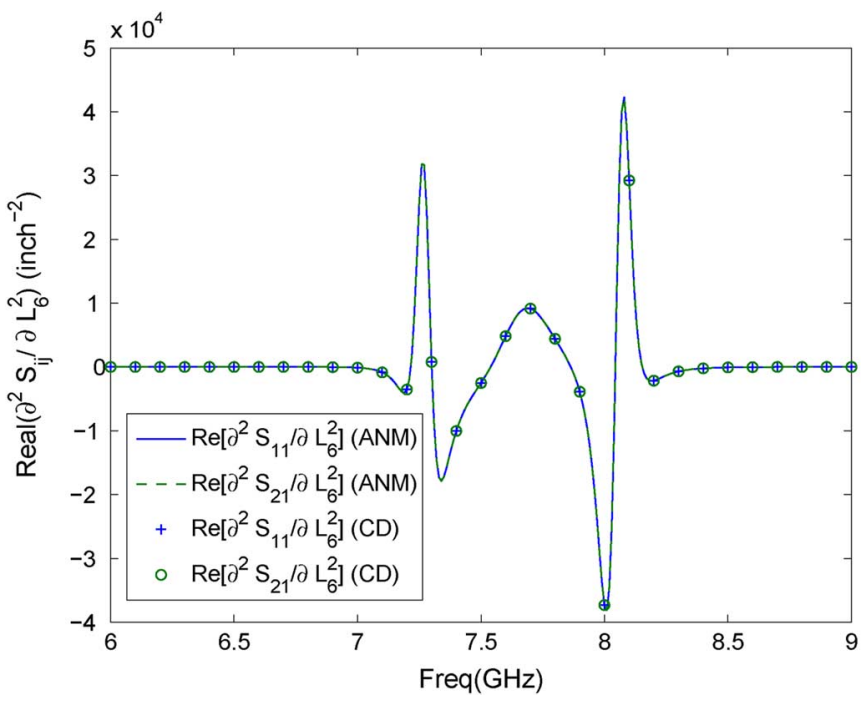

Fig. 7. Real part of the dominant mode scattering parameters secondorder derivatives w.r.t. $L_{6}$ of the five-pole filter with center frequency $f_{0}=7.66 \mathrm{GHz}$, obtained using the ANM and finite CDs. (Color version available online at: http://ieeexplore.ieee.org.)



Fig. 8. Imaginary part of the dominant mode scattering parameters secondorder derivatives w.r.t. $L_{1}$ of the five-pole filter with center frequency $f_{0}=$ $7.66 \mathrm{GHz}$, obtained using the ANM and finite CDs. (Color version available online at: http://ieeexplore.ieee.org.)

duces a sufficient decrease in the residuals of $\boldsymbol{r}$ at the new point $\boldsymbol{x}_{k}+\boldsymbol{h}_{k}$. It then sets $\boldsymbol{x}_{k+1}=\boldsymbol{x}_{k}+\boldsymbol{h}_{k}$ and begins a new iteration with $\boldsymbol{x}_{k+1}$ replacing $\boldsymbol{x}_{k}$. A sufficient decrease in the residuals implies that $f\left(\boldsymbol{x}_{k}+\boldsymbol{h}_{k}\right)<f\left(\boldsymbol{x}_{k}\right)$. The correction $\boldsymbol{h}_{k}$ depends upon a damping parameter, the residual $\boldsymbol{r}$ and its Jacobian at $\boldsymbol{x}_{k}$. The optimization step $h_{k}$ is defined as [1]

$$
\left(\nabla^{2} f(\boldsymbol{x})+\boldsymbol{\mu} \mathbf{I}\right) \boldsymbol{h}_{k}=-\mathbf{J}^{T} \boldsymbol{r},
$$

where $\boldsymbol{\mu}$ is a diagonal matrix of damping parameters. During iterations, the value of $\boldsymbol{\mu}$ is controlled by the gain ratio

$$
\rho=\frac{f\left(\boldsymbol{x}_{k}\right)-f\left(\boldsymbol{x}_{k}+\boldsymbol{h}_{k}\right)}{q\left(\boldsymbol{x}_{k}\right)-q\left(\boldsymbol{x}_{k}+\boldsymbol{h}_{k}\right)}
$$




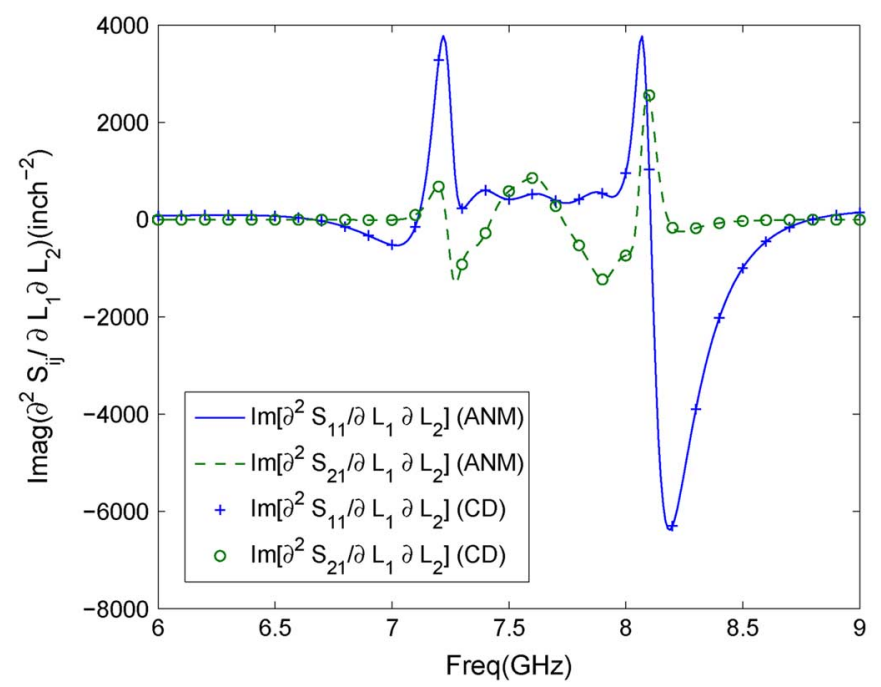

Fig. 9. Imaginary part of the dominant mode scattering parameters secondorder derivatives w.r.t. $L_{1}$ and $L_{2}$ of the five-pole filter with center frequency $f_{0}=7.66 \mathrm{GHz}$, obtained using the ANM and finite CDs. (Color version available online at: http://ieeexplore.ieee.org.)

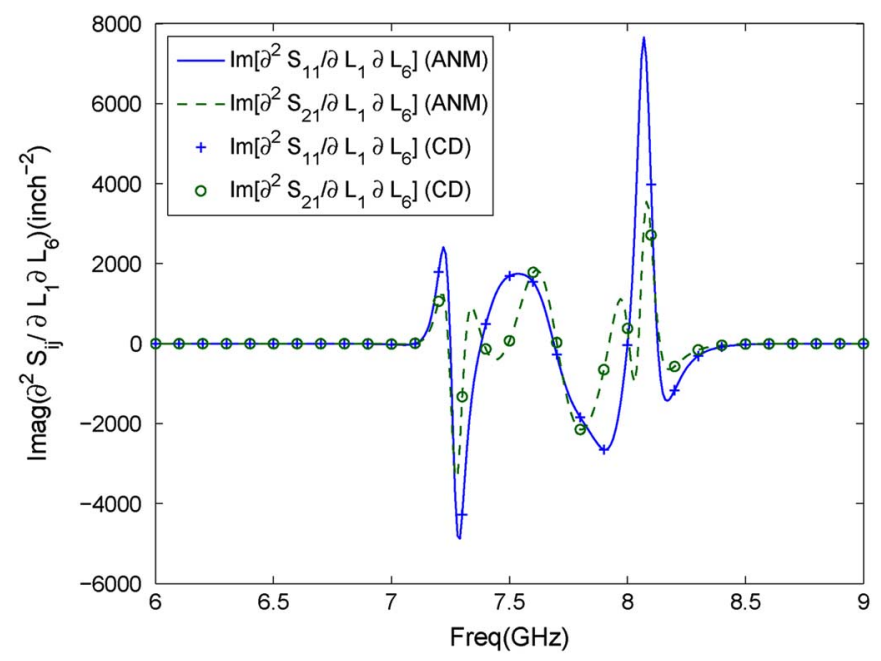

Fig. 10. Imaginary part of the dominant mode scattering parameters secondorder derivatives w.r.t. $L_{1}$ and $L_{6}$ of the five-pole filter with center frequency $f_{0}=7.66 \mathrm{GHz}$, obtained using the ANM and finite CDs. (Color version available online at: http://ieeexplore.ieee.org.)

as follows:

and

$$
\boldsymbol{\mu}= \begin{cases}2 \boldsymbol{\mu}, & \rho<0.25 \\ \frac{1}{2} \boldsymbol{\mu}, & \rho>0.75\end{cases}
$$

$$
q\left(\boldsymbol{x}_{k}\right)-q\left(\boldsymbol{x}_{k}+\boldsymbol{h}_{k}\right)=-\boldsymbol{h}_{k}^{T} \mathbf{J}^{T} \boldsymbol{r}-\frac{1}{2} \boldsymbol{h}_{k}^{T} \nabla^{2} f\left(\boldsymbol{x}_{k}\right) \boldsymbol{h}_{k} .
$$

Although the value of $\boldsymbol{\mu}$ is controlled by the gain ratio, its initial value can be critical in determining the number of iterations and the optimization time.

\section{NumericAl RESUlTS AND Discussion}

A five-pole ridge waveguide filter (see Fig. 2) is first designed, using full-wave modeling, to have a center frequency $f_{0}=7.66 \mathrm{GHz}$ and a bandwidth $\mathrm{BW}=0.8 \mathrm{GHz}$. The filter has

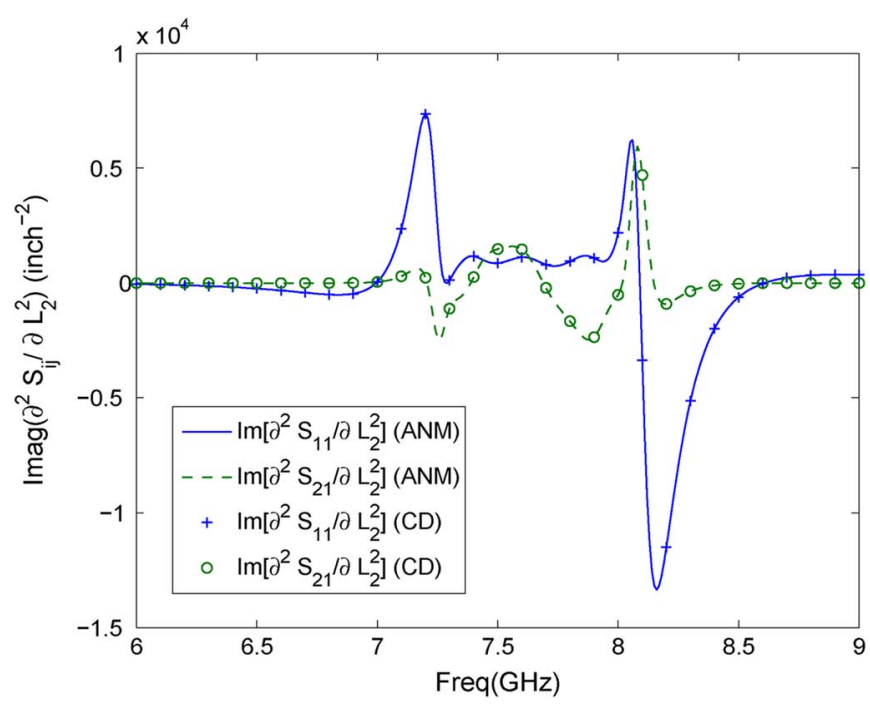

Fig. 11. Imaginary part of the dominant mode scattering parameters secondorder derivatives w.r.t. $L_{2}$ of the five-pole filter with center frequency $f_{0}=$ $7.66 \mathrm{GHz}$, obtained using the ANM and finite CDs. (Color version available online at: http://ieeexplore.ieee.org.)

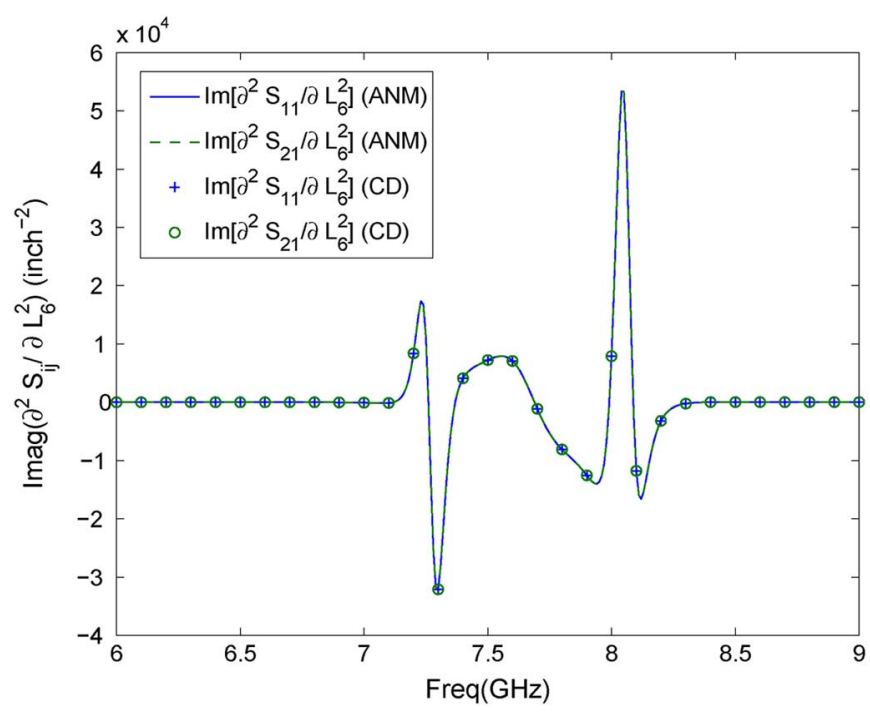

Fig. 12. Imaginary part of the dominant mode scattering parameters secondorder derivatives w.r.t. $L_{6}$ of the five-pole filter with center frequency $f_{0}=$ $7.66 \mathrm{GHz}$, obtained using the ANM and finite CDs. (Color version available online at: http://ieeexplore.ieee.org.)

the initial response shown in Fig. 2. The passband return loss $(12.6 \mathrm{~dB})$, the center frequency $(7.8425 \mathrm{GHz})$, and the bandwidth $(0.863 \mathrm{GHz})$ do not satisfy the specifications as required in Table I. The filter response is obtained using the rigorous MM technique. The number of modes used to characterize each ridge to rectangular waveguide discontinuity is 12 (eight TE modes and four TM modes). This number of modes satisfies convergence, as indicated in [24]. Results obtained from Ansoft's High Frequency Structure Simulator (HFSS) [25] are compared to those computed using the MM technique. Both results are in very good agreement, which validates the accuracy of the developed in-house MM simulator. The vector of designable parameters is $\boldsymbol{x}=\left[\begin{array}{llll}L_{1} & L_{2} & \cdots & L_{11}\end{array}\right]^{T} \in \mathbb{R}^{11 \times 1}$. The initial value of $\boldsymbol{x}$ is indicated in Table II. Figs. 3-7 and Figs. 8-12 show the real and 


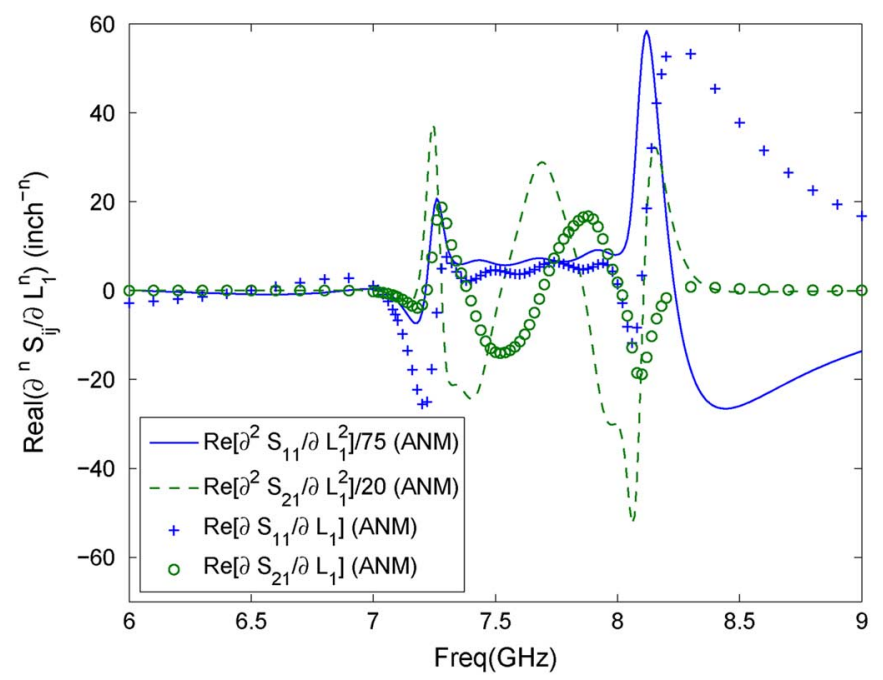

Fig. 13. Real part of the dominant mode scattering parameters first- and second-order derivatives w.r.t. $L_{1}$ of the five-pole filter with center frequency $f_{0}=7.66 \mathrm{GHz}$, obtained using the ANM. The displayed values of the real part of the second-order derivatives of $S_{11}$ and $S_{21}$ were divided by 75 and 20, respectively, for better display of the curves. (Color version available online at: http://ieeexplore.ieee.org.)

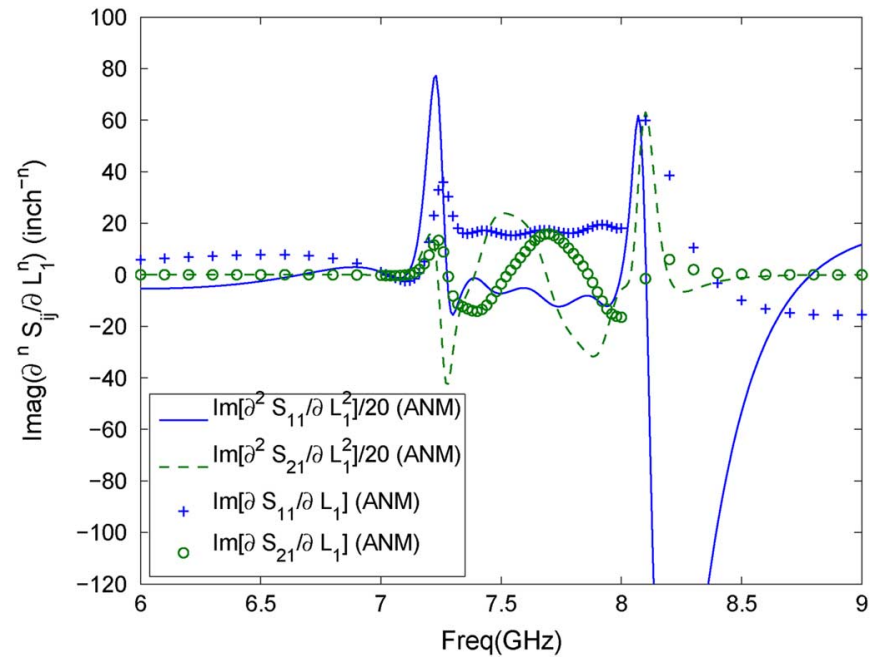

Fig. 14. Imaginary part of the dominant mode scattering parameters first- and second-order derivatives w.r.t. $L_{1}$ of the five-pole filter with center frequency $f_{0}=7.66 \mathrm{GHz}$, obtained using the ANM. The displayed values of the real part of the second-order derivatives of $S_{11}$ and $S_{21}$ were divided by 20 for better display of the curves. (Color version available online at: http://ieeexplore.ieee. org.)

imaginary parts, respectively, of the second-order derivatives of the dominant mode scattering parameters w.r.t. the design parameters. It should be noted that in Figs. 7 and 12,

$$
\frac{\partial^{2} S_{11}}{\partial L_{6}^{2}}=\frac{\partial^{2} S_{21}}{\partial L_{6}^{2}} .
$$

Figs. 13 and 14 show the real and imaginary parts, respectively, of the dominant mode scattering parameters first- and secondorder derivatives w.r.t. $L_{1}$. These figures show the following. $\partial\left|S_{11}\right| / \partial L_{1}=0$ and $\partial^{2}\left|S_{11}\right| / \partial L_{1}^{2}>0$ at the passband edges so the minimum of $\left|S_{11}\right|$ (in absolute value) occurs at the edges of the passband. The first order derivative has no other zeros.



Fig. 15. Optimized responses of the five-pole filter with center frequency $f_{0}=$ $7.66 \mathrm{GHz}$ obtained using BFGS and LM methods compared to HFSS (using the LM dimensions). The optimized dimensions using BFGS and LM are shown in Table II. (Color version available online at: http://ieeexplore.ieee.org.)

Thus, $\left|S_{11}\right|$ has no other peak within the passband and it remains below the specified return loss in the entire passband. Similarly, the minimum of $\left|S_{21}\right|$ (in absolute value) occurs at the edges of the passband and then alternates between maximum and minimum due to ripples in the transmission within the passband. The results in Figs. 13 and 14 are typical for a good designed filter where the main objective is to minimize $\left|S_{11}\right|$ and to maximize $\left|S_{21}\right|$ in the passband. In Figs. 3-14, the utilized values of the design parameters are those obtained from the LM optimization, as indicated in Table II. All results obtained using the ANM are compared to those obtained from CDs, and they are in excellent agreement. The formulas used for CDs are based on function calls only and are given by [15]

$$
\begin{array}{r}
\frac{\partial^{2} f}{\partial x_{i}^{2}}=\frac{1}{12 h_{i}^{2}}(-f(\boldsymbol{x} \\
\left.+2 h_{i} \boldsymbol{e}_{i}\right)-f\left(\boldsymbol{x}-2 h_{i} \boldsymbol{e}_{i}\right)-30 f(\boldsymbol{x}) \\
\left.+16 f\left(\boldsymbol{x}+h_{i} \boldsymbol{e}_{i}\right)+16 f\left(\boldsymbol{x}-h_{i} \boldsymbol{e}_{i}\right)\right)
\end{array}
$$

and

$$
\begin{aligned}
\frac{\partial^{2} f}{\partial x_{i} \partial x_{j}}= & \frac{1}{4 h_{i} h_{j}}\left(f\left(\boldsymbol{x}+h_{i} \boldsymbol{e}_{i}+h_{j} \boldsymbol{e}_{j}\right)\right. \\
& -f\left(\boldsymbol{x}+h_{i} \boldsymbol{e}_{i}-h_{j} \boldsymbol{e}_{j}\right)-f\left(\boldsymbol{x}-h_{i} \boldsymbol{e}_{i}+h_{j} \boldsymbol{e}_{j}\right) \\
& \left.+f\left(\boldsymbol{x}-h_{i} \boldsymbol{e}_{i}-h_{j} \boldsymbol{e}_{j}\right)\right)
\end{aligned}
$$

where $f \in\left\{S_{11}, S_{21}\right\}$, subscripts $i, j \in\{1,2, \ldots, n\}, \boldsymbol{x}=$ $\left[\begin{array}{llll}x_{1} & x_{2} & \cdots & x_{n}\end{array}\right]^{T}$ is the vector of design parameters, and $\boldsymbol{e}_{i}=$

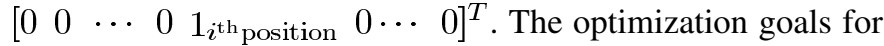
the implemented optimization routines (BFGS and LM) are as shown in Table I. The BFGS method gives the following results:

$$
\begin{aligned}
\# \text { of iterations } & =68 \\
\# \text { of function evaluations } & =208
\end{aligned}
$$

\# of segments explored during Cauchy searching $=74$

Optimization time $=2419 \mathrm{~s}$. 


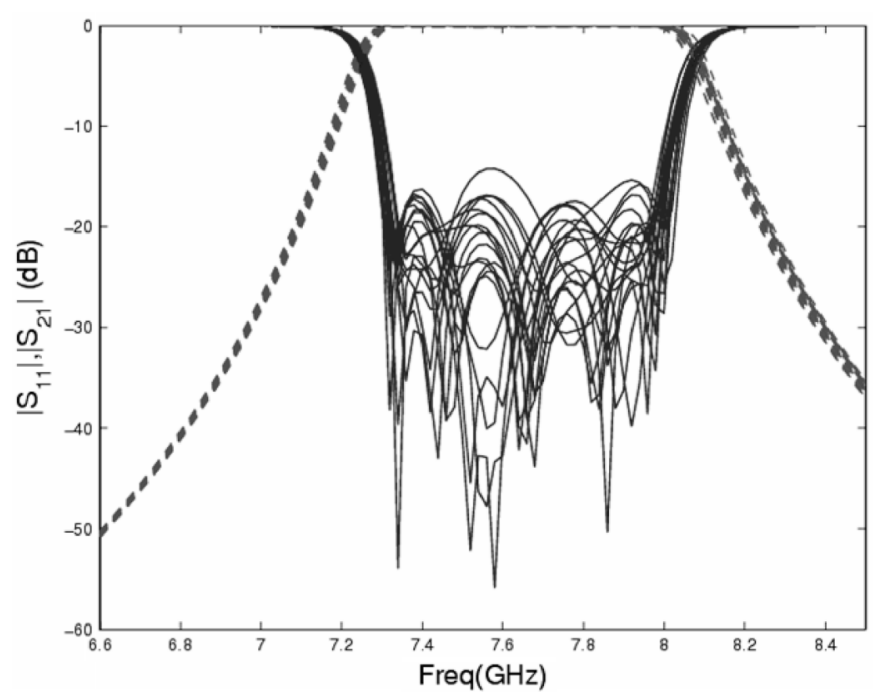

(a)

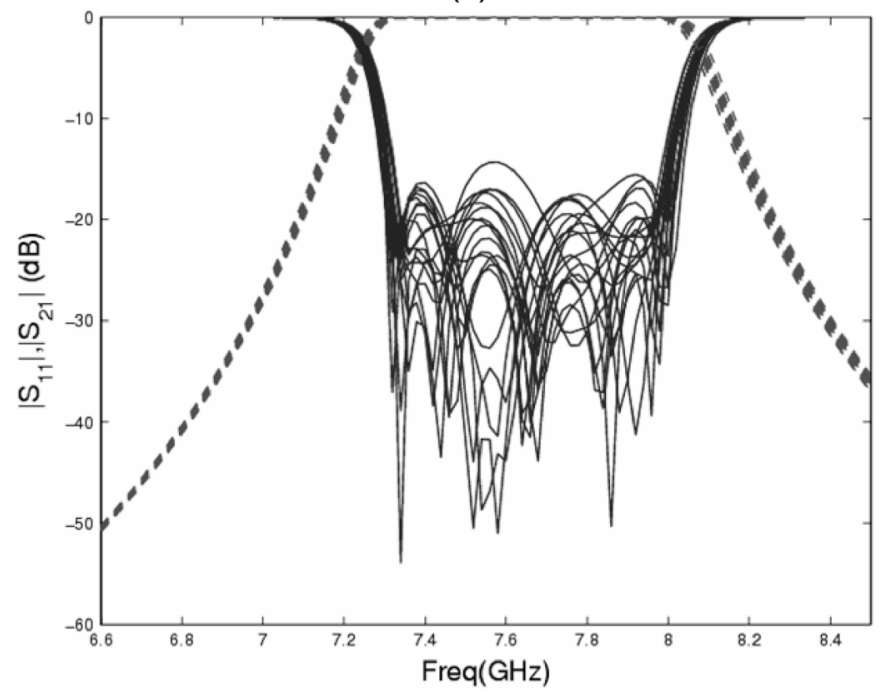

(b)

Fig. 16. Tolerance analysis assuming \pm 1 -mil statistical variation of the dimensions $L_{1}$ to $L_{11}$ of the five-pole filter with center frequency $f_{0}=7.66 \mathrm{GHz}$. (a) Responses are obtained using first- and second-order derivatives. (b) Responses are obtained from direct MM simulation.

The LM method (initial value of each element of $\boldsymbol{\mu}$ is the norm of the corresponding column of the initial Hessian matrix) gives the following results:

$$
\begin{aligned}
\# \text { of iterations } & =23 \\
\# \text { of function evaluations } & =23 \\
\# \text { of gradient evaluations } & =20 \\
\# \text { of Hessian evaluations } & =20 \\
\text { Optimization time } & =524 \mathrm{~s} .
\end{aligned}
$$

The optimized vector of design parameters $\boldsymbol{x}$ has the dimensions shown in Table II. The filter is symmetric around $L_{6}$. The computation time for the BFGS method is approximately $5 \times$ that of the LM method. The simulations, obtained from a developed FORTRAN code, were run on a dual 750-MHz UltraSPARC III processors with $1 \mathrm{~GB}$ of memory and running SunOS 5.8 (i.e., Solaris 8). The savings in time using the LM method is

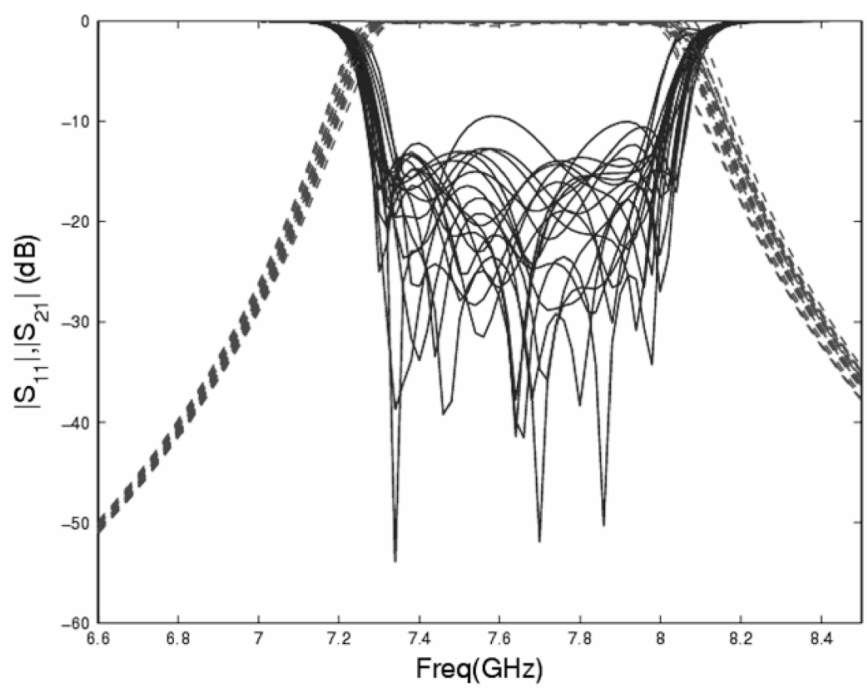

(a)

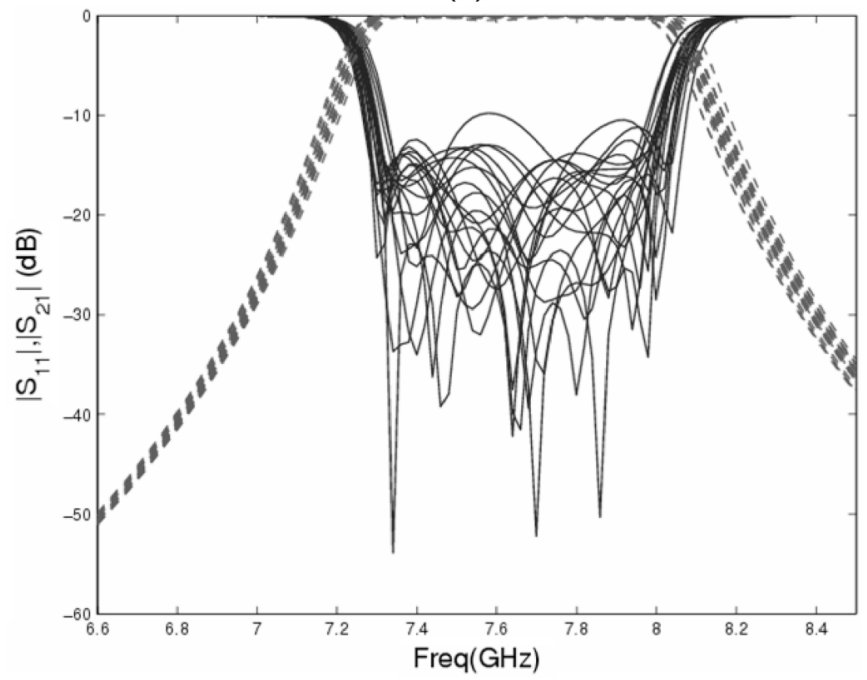

(b)

Fig. 17. Tolerance analysis assuming \pm 2 -mil statistical variation of the dimensions $L_{1}$ to $L_{11}$ of the five-pole filter with center frequency $f_{0}=7.66 \mathrm{GHz}$. (a) Responses are obtained using first- and second-order derivatives. (b) Responses are obtained from direct MM simulation.

due to the sparsity of the matrices in (10). This makes the computation of the Hessian matrix in (24) very fast.

Comparison between the responses obtained from both methods and HFSS using the LM optimized lengths is shown in Fig. 15. The results are similar, which shows the advantage of the LM method over the BFGS method regarding computation time. The return loss obtained from the BFGS optimization is better than the one obtained from the LM routine. However, the selectivity obtained from the LM is better than the one obtained from the BFGS algorithm and the bandwidth obtained from the LM fits exactly the specifications.

Tolerance analysis for $\pm 1 \mathrm{mil}(25.4 \mu \mathrm{m}), \pm 2 \mathrm{mil}(50.8 \mu \mathrm{m})$, and $\pm 5 \mathrm{mil}(127 \mu \mathrm{m})$ statistical variation of the dimensions $L_{1}$ to $L_{11}$ are shown in Figs. 16-18, respectively. Figs. 16(a), 17(a), and 18(a) show the tolerance analysis obtained using the firstand second-order derivative information

$$
f(\boldsymbol{x}+\boldsymbol{h}) \approx f(\boldsymbol{x})+\boldsymbol{h}^{T} \nabla f(\boldsymbol{x})+\frac{1}{2} \boldsymbol{h}^{T} \nabla^{2} f(\boldsymbol{x}) \boldsymbol{h}
$$




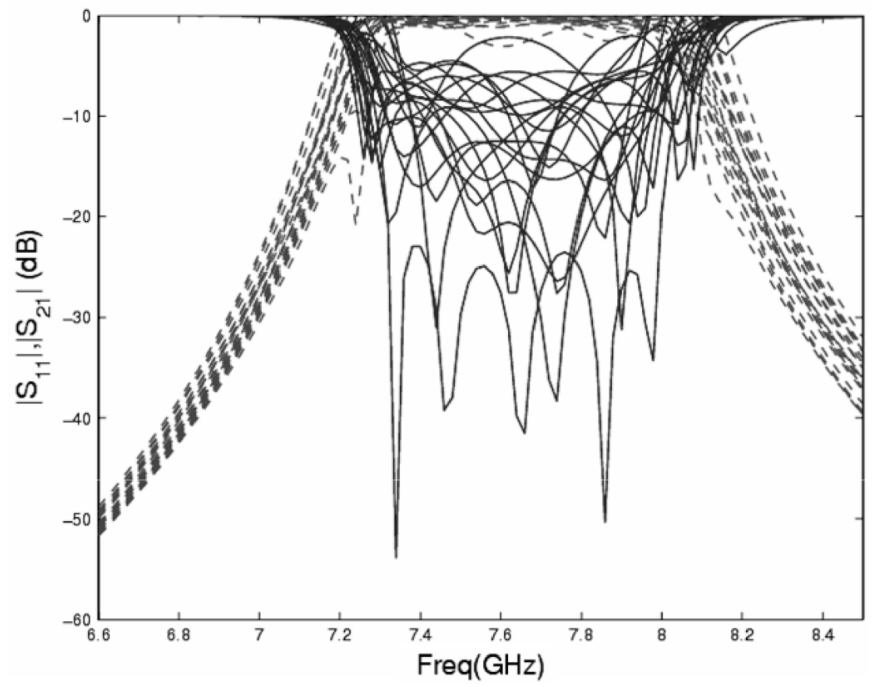

(a)

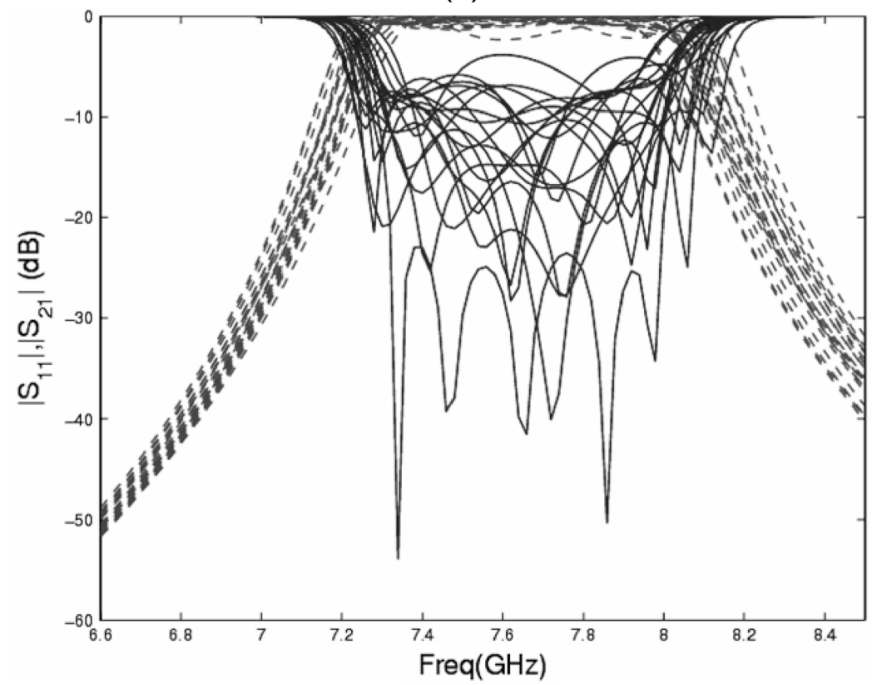

(b)

Fig. 18. Tolerance analysis assuming \pm 5 -mil statistical variation of the dimensions $L_{1}$ to $L_{11}$ of the five-pole filter with center frequency $f_{0}=7.66 \mathrm{GHz}$. (a) Responses are obtained using first- and second-order derivatives. (b) Responses are obtained from direct MM simulation.

TABLE III

DIMENSIONS OF $\boldsymbol{x}$ (IN INCHES) BEFORE AND AFTER OPTIMIZATION FOR THE Ridge WaVEGUide Filter With CENTER FREQUENCY $f_{0}=9.89 \mathrm{GHz}$ $L_{7}=L_{5}, L_{8}=L_{4}, L_{9}=L_{3}, L_{10}=L_{2}, L_{11}=L_{1}$

\begin{tabular}{|l|c|c|c|c|c|c|}
\hline & $L_{1}$ & $L_{2}$ & $L_{3}$ & $L_{4}$ & $L_{5}$ & $L_{6}$ \\
\hline Initial & 0.0415 & 0.0378 & 0.1151 & 0.0180 & 0.1436 & 0.0164 \\
\hline BFGS & 0.0405 & 0.0387 & 0.1182 & 0.018 & 0.1535 & 0.0162 \\
\hline LM & 0.0402 & 0.0383 & 0.1143 & 0.0179 & 0.1481 & 0.0160 \\
\hline
\end{tabular}

where $\boldsymbol{h}$ is the perturbation of $\boldsymbol{x}$ from its nominal value. Figs. 16(b), 17(b), and 18(b) show the tolerance analysis obtained using direct MM simulation. These figures show that up to a manufacturing tolerance of \pm 2 mil, the responses are good. The responses obtained from the Taylor expansion (38) also agree very well with the responses obtained from direct MM simulations. For a maximum tolerance varying from 1 to $5 \mathrm{mil}$, the corresponding maximum relative perturbation percentage

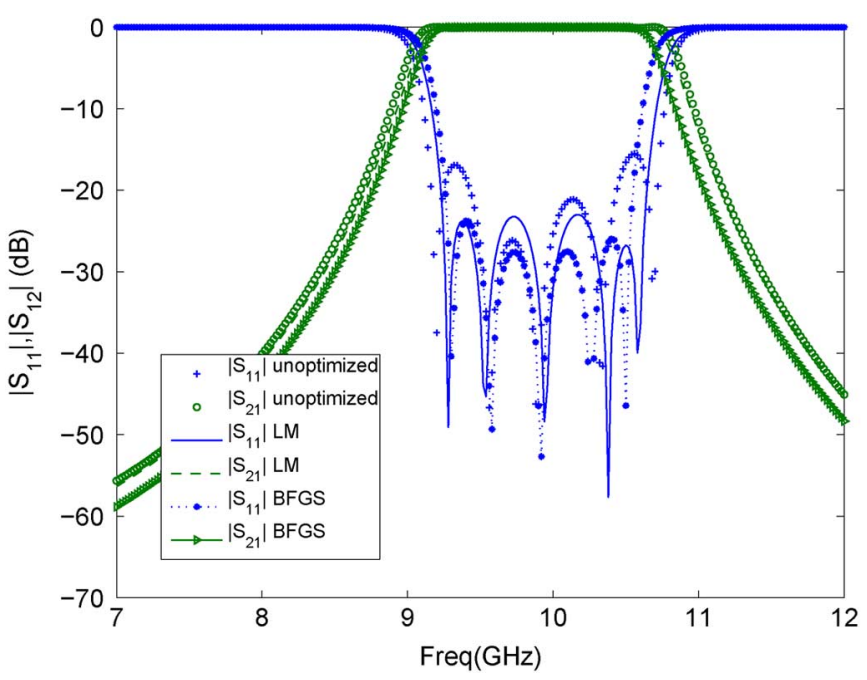

Fig. 19. Optimized responses obtained using BFGS and LM methods compared to the nonoptimized response for the five-pole filter with center frequency $f_{0}=9.89 \mathrm{GHz}$. Waveguide cross section: 0.18 in $\times 0.071$ in, ridge width $w=0.08$ in, ridge gap $=0.0138 \mathrm{in}, \varepsilon_{r}=5.9, L_{1}$ to $L_{11}$ initial and optimized using BFGS and LM are shown in Table III. (Color version available online at: http://ieeexplore.ieee.org.)

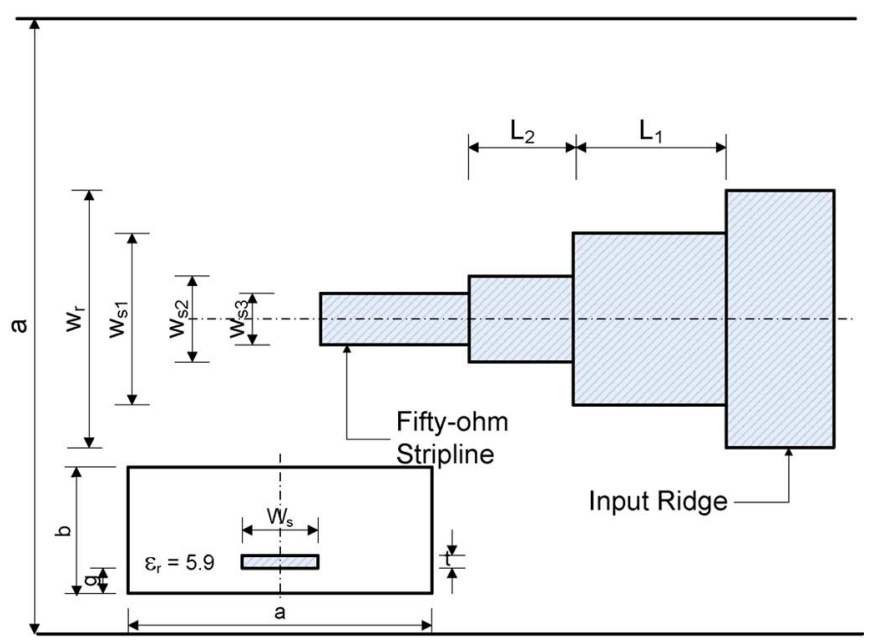

Fig. 20. Top view of the transition from the input ridge waveguide to a $50-\Omega$ stripline. The inset shows the cross section of the stripline $a=0.24$ in, $b=0.071 \mathrm{in}, g=0.0138 \mathrm{in}, w_{r}=0.08 \mathrm{in}, w_{s 1}=$ $0.04 \mathrm{in}, w_{s 2}=0.082 \mathrm{in}, w_{s 3}=0.0135 \mathrm{in}$. (Color version available online at: http://ieeexplore.ieee.org.)

$\left(P_{\max } \%\right)$ varies from $0.26 \%$ to $1.32 \%$. The maximum relative perturbation is defined as

$$
P_{\max }=\frac{\max \left(\|\boldsymbol{h}\|_{2}\right)}{\|\boldsymbol{x}\|_{2}}=\frac{\operatorname{tol}_{\max } \times \sqrt{n}}{\|\boldsymbol{x}\|_{2}}
$$

where it is assumed that the $n$ design variables are all perturbed by the maximum manufacturing tolerance $\left( \pm\right.$ tol $\left._{\max }\right)$. Actually, this tolerance variation corresponds from $2.8 \%$ to $14 \%$ of the middle resonator length $\left(L_{6}\right)$, while it represents from $0.41 \%$ to $2 \%$ of the middle evanescent sections length $\left(L_{5}, L_{7}\right)$. In Figs. 16-18, and 20 perturbed responses are run for each manufacturing tolerance. Each response has 100 frequency points. 


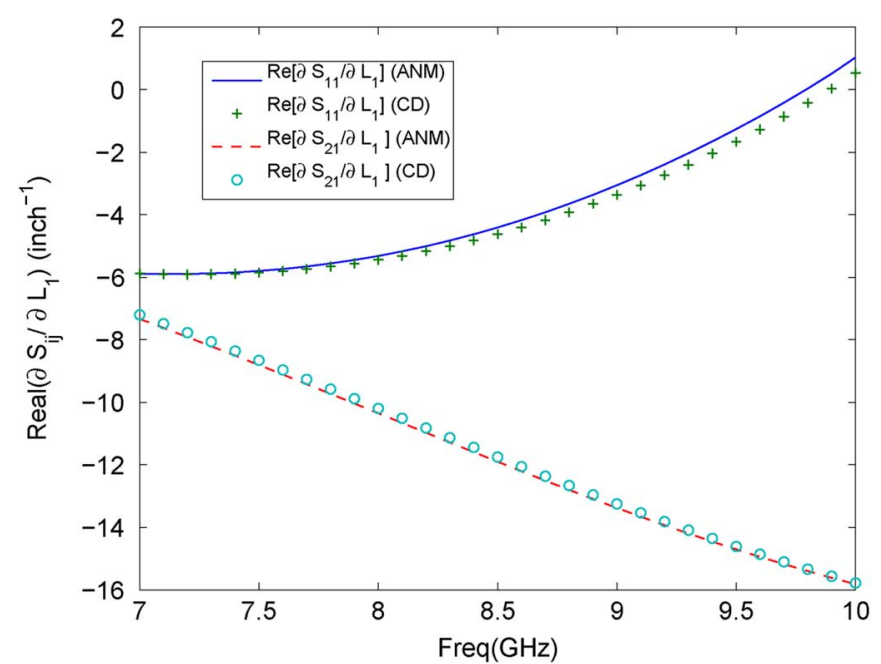

Fig. 21. Real part of the dominant mode scattering parameters sensitivity w.r.t. $L_{1}$ of the transition, obtained using the ANM and finite CDs. (Color version available online at: http://ieeexplore.ieee.org.)

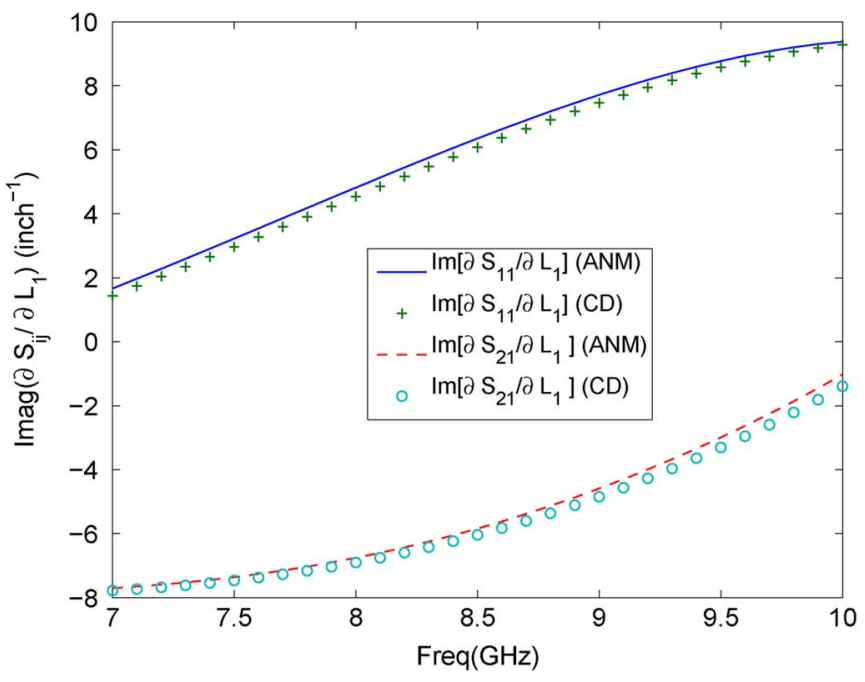

Fig. 22. Imaginary part of the dominant mode scattering parameters sensitivity w.r.t. $L_{1}$ of the transition, obtained using the ANM and finite CDs. (Color version available online at: http://ieeexplore.ieee.org.)

The time required by direct MM simulation is $286 \mathrm{~s}$. The Taylor approximation, on the other hand, required only $103 \mathrm{~s}$.

A second optimization example for a five-pole ridge waveguide filter with a center frequency $f_{0}=9.89 \mathrm{GHz}$, passband ripple $0.01 \mathrm{~dB}$, and equiripple bandwidth from 9.23 to $10.63 \mathrm{GHz}$ is carried out. The BFGS method gives the following results:

$$
\begin{aligned}
\# \text { of iterations } & =55 \\
\text { Optimization time } & =2171 \mathrm{~s} .
\end{aligned}
$$

The LM method gives the following results:

$$
\begin{aligned}
\# \text { of iterations } & =24 \\
\# \text { of gradient evaluations } & =7 \\
\# \text { of Hessian evaluations } & =7 \\
\text { Optimization time } & =508 \mathrm{~s} .
\end{aligned}
$$

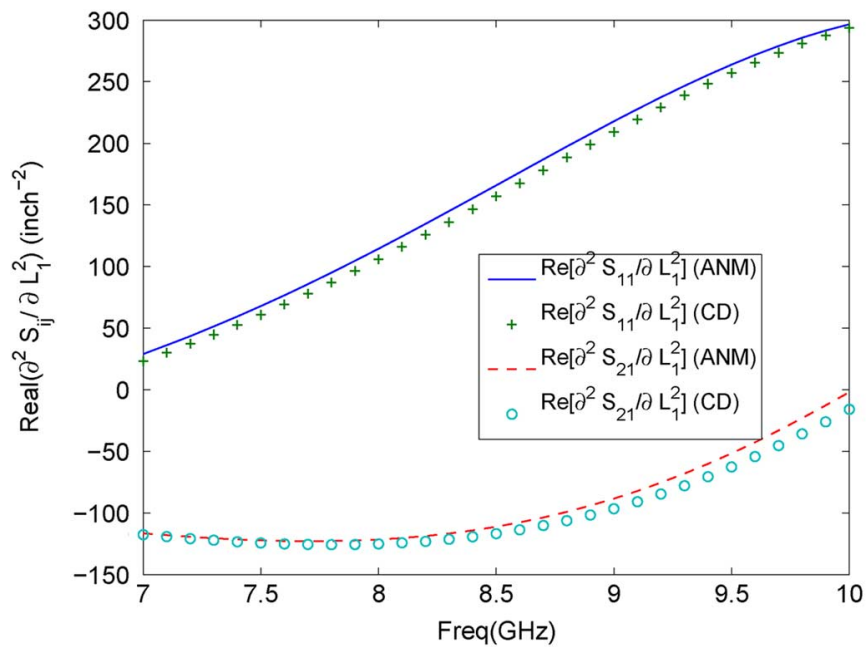

Fig. 23. Real part of the dominant mode scattering parameters second-order derivatives w.r.t. $L_{1}$ of the transition, obtained using the ANM and finite CDs. (Color version available online at: http://ieeexplore.ieee.org.)

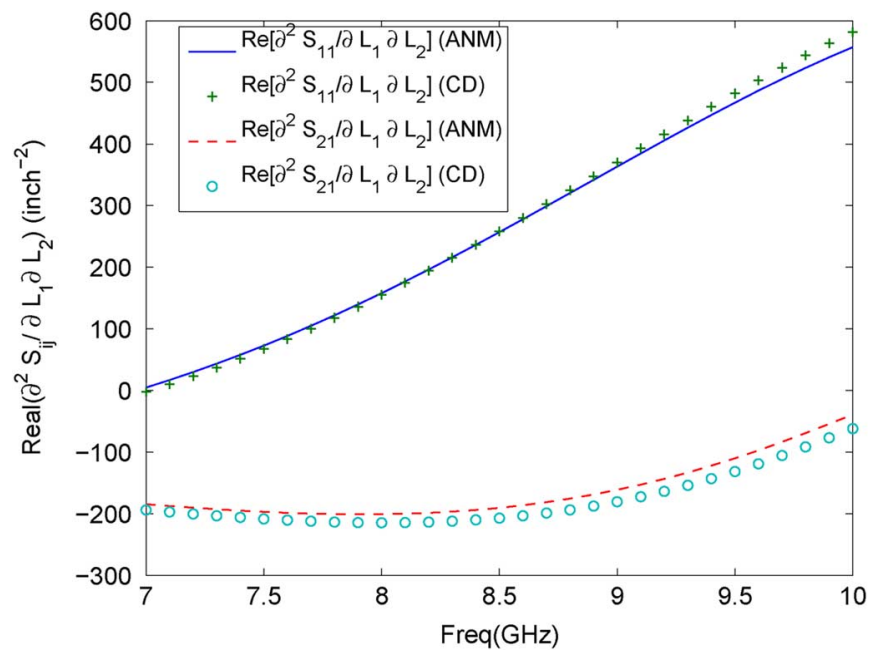

Fig. 24. Real part of the dominant mode scattering parameters second-order derivatives w.r.t. $L_{1}$ and $L_{2}$ of the transition, obtained using the ANM and finite CDs. (Color version available online at: http://ieeexplore.ieee.org.)

The optimized vector of design parameters $x$ has the dimensions shown in Table III. The computation time for the BFGS method is more than $4 \times$ that of the LM method. The optimized responses compared to the nonoptimized response are shown in Fig. 19.

The developed optimization technique is also applied to a third example. The transition from the input/output ridge waveguide to a $50-\Omega$ stripline, shown in Fig. 20, is optimized w.r.t. the lengths $L_{1}$ and $L_{2}$. The number of modes used to characterize each stripline to stripline discontinuity is 15 (ten TE modes and five TM modes). The real and imaginary parts of the first- and second-order derivatives are shown in Figs. 21-26. Using the obtained first- and second-order derivatives, the transition is optimized over the frequency range of $7-10 \mathrm{GHz}$ to 


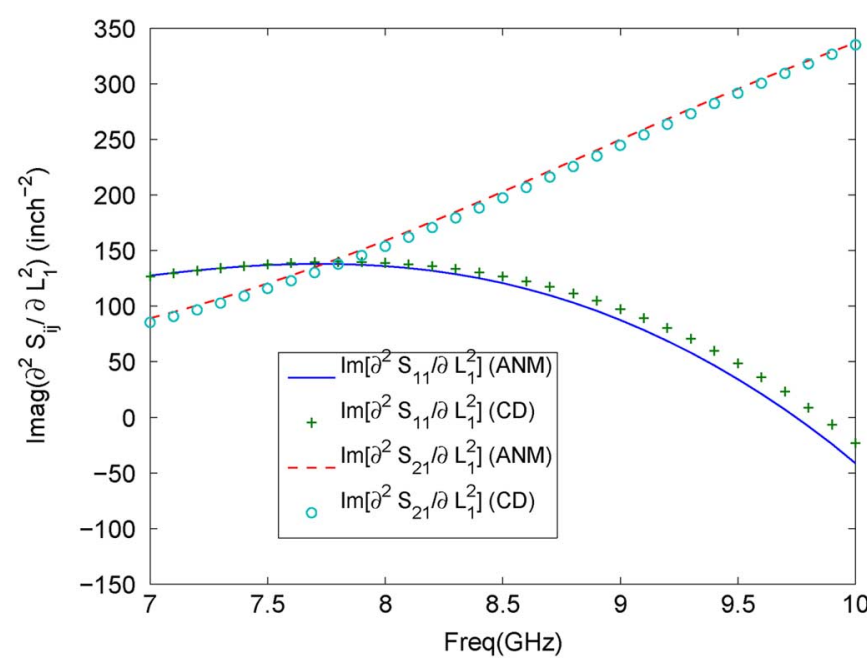

Fig. 25. Imaginary part of the dominant mode scattering parameters secondorder derivatives w.r.t. $L_{1}$ of the transition, obtained using the ANM and finite CDs. (Color version available online at: http://ieeexplore.ieee.org.)

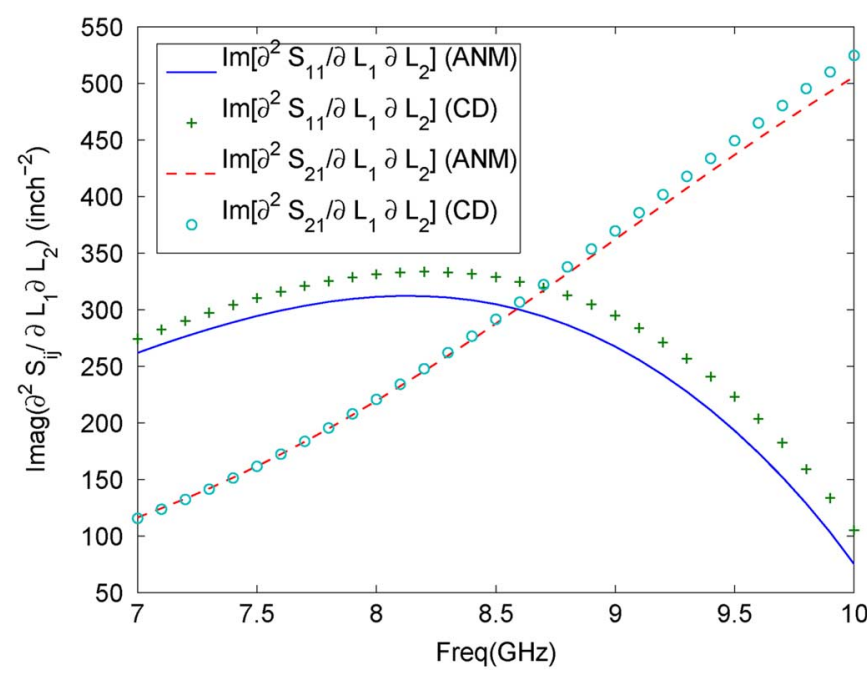

Fig. 26. Imaginary part of the dominant mode scattering parameters secondorder derivatives w.r.t. $L_{1}$ and $L_{2}$ of the transition, obtained using the ANM and finite CDs. (Color version available online at: http://ieeexplore.ieee.org.)

satisfy a return loss of 0.01 . The BFGS method gives the following results:

$$
\begin{aligned}
\# \text { of function evaluations } & =34 \\
\text { \#of segments explored during Cauchy searching } & =10 \\
\text { Optimization time } & =318 \mathrm{~s} .
\end{aligned}
$$

The LM method gives the following results:

$$
\begin{aligned}
\# \text { of function evaluations } & =10 \\
\# \text { of gradient evaluations } & =7 \\
\# \text { of Hessian evaluations } & =7 \\
\text { Optimization time } & =98 \mathrm{~s} .
\end{aligned}
$$

TABLE IV

DIMENSIONS OF $\boldsymbol{x}$ (IN INCHES) BEFORE AND AFTER OPTIMIZATION FOR THE RIDGE WAVEGUIDE TO $50-\Omega$ STRIPLINE TRANSITION

\begin{tabular}{|l|c|c|}
\hline & $L_{s 1}$ & $L_{s 2}$ \\
\hline Initial & 0.1 & 0.03 \\
\hline BFGS & 0.097 & 0.008 \\
\hline LM & 0.095 & 0.008 \\
\hline
\end{tabular}

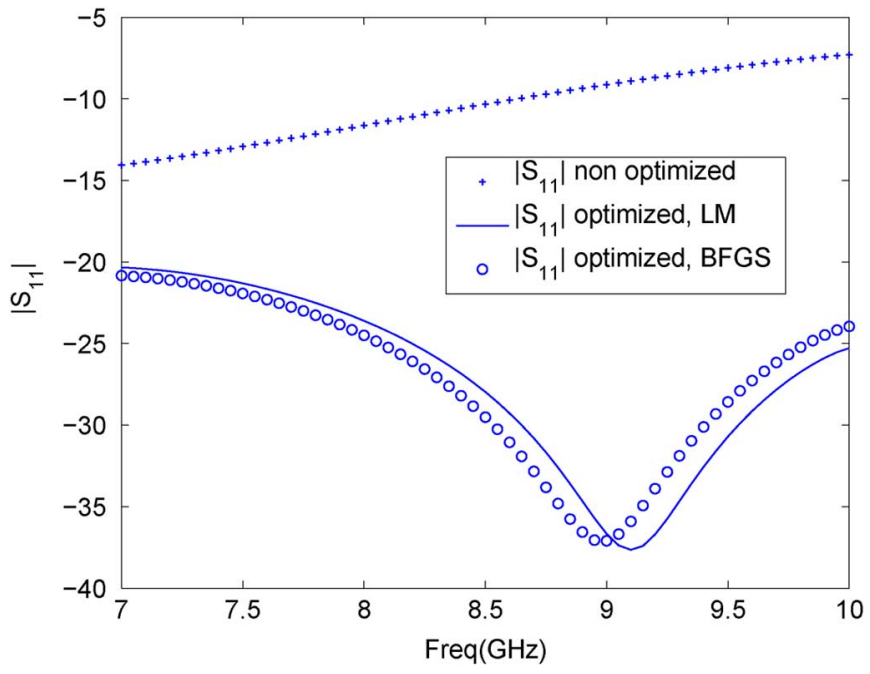

Fig. 27. Optimized responses of the transition obtained using BFGS and LM methods compared to the nonoptimized. The optimized dimensions using BFGS and LM are shown in Table IV. (Color version available online at: http://ieeexplore.ieee.org.)

The optimized vector of design parameters $x$ for the transition has the dimensions shown in Table IV. The computation time for the BFGS method is more than $3 \times$ that of the LM method. Comparison between the initial response and those obtained from both methods is shown in Fig. 27. The results are similar, which again shows the advantage of the LM method over the BFGS method regarding computation time.

\section{CONCLUSION}

The ANM has been applied to estimate the sensitivities of scattering parameters of microwave filters and transitions, obtained with the full-wave MM technique. Using only the MM simulation of the original network, the first- and second-order sensitivities of the scattering parameters w.r.t. all the designable parameters have been obtained. The higher order modes (propagating and evanescent) characterizing the discontinuities have been considered for better accuracy. The obtained sensitivities (first- and second-order derivatives) have been used to compute the gradient and the Hessian matrices of differentiable objective functions. The formulation has been applied to the sensitivity analysis of ridge waveguide filters and transitions from ridge waveguides to $50-\Omega$ striplines. Our optimization routine utilizing analytical Hessian matrices is faster than the BFGS routine using only the analytical gradient. Statistical analysis obtained using an adjoint-based approximate model is accurate and much faster than direct MM simulation. 


\section{APPENDIX \\ DERIVATION OF (27)}

Let the magnitude of the complex scattering parameter be represented as

$$
\left|S_{i j}\right|=\sqrt{a^{2}+b^{2}}
$$

where $a$ and $b$ are the real and imaginary parts of the complex scattering parameter, respectively. Taking the derivatives of both sides of (A-1), the derivative of the magnitude is given as

$$
\left|S_{i j}\right| \frac{\partial\left|S_{i j}\right|}{\partial x}=\left(a \frac{\partial a}{\partial x}+b \frac{\partial b}{\partial x}\right) .
$$

Taking the derivative of (A-2) w.r.t. $y$,

$$
\begin{aligned}
\frac{\partial\left|S_{i j}\right|}{\partial x} \frac{\partial\left|S_{i j}\right|}{\partial y}+\left|S_{i j}\right| \frac{\partial^{2}\left|S_{i j}\right|}{\partial x \partial y}= & \frac{\partial a}{\partial x} \frac{\partial a}{\partial y}+\frac{\partial b}{\partial x} \frac{\partial b}{\partial y} \\
& +a \frac{\partial^{2} a}{\partial x \partial y}+b \frac{\partial^{2} b}{\partial x} \partial y .
\end{aligned}
$$

The following relations can also be easily proven:

$$
\begin{aligned}
\frac{\partial a}{\partial x} \frac{\partial a}{\partial y}+\frac{\partial b}{\partial x} \frac{\partial b}{\partial y} & =\operatorname{Re}\left(\frac{\partial S_{i j}}{\partial x} \frac{\partial S_{i j}^{*}}{\partial y}\right) \\
a \frac{\partial^{2} a}{\partial x \partial y}+b \frac{\partial^{2} b}{\partial x} \partial y & =\operatorname{Re}\left(S_{i j}^{*} \frac{\partial^{2} S_{i j}}{\partial x \partial y}\right)
\end{aligned}
$$

where $*$ denotes the conjugate. Substituting from (A-4) into (A-3) and doing simple manipulations, (27) is obtained.

\section{REFERENCES}

[1] J. E. Dennis, Jr. and R. B. Schnabel, Numerical Methods for Unconstrained Optimization and Nonlinear Equations. Englewood Cliffs, NJ: Prentice-Hall, 1983.

[2] E. S. Kuh and C. G. Lau, "Sensitivity invariants of continuously equivalent networks," IEEE Trans. Circuit Theory, vol. CT-15, no. 9, pp. 175-177, Sep. 1968.

[3] S. W. Director and R. A. Rohrer, "The generalized adjoint network and network sensitivities," IEEE Trans. Circuit Theory, vol. CT-16, no. 8, pp. 318-323, Aug. 1969.

[4] M. Sablatash and R. Seviora, "Sensitivity invariants for scattering matrices," IEEE Trans. Circuit Theory, vol. CT-18, no. 3, pp. 282-284, Mar. 1971.

[5] J. W. Bandler and R. E. Seviora, "Wave sensitivities of networks," IEEE Trans. Microw. Theory Tech., vol. MTT-20, no. 2, pp. 138-147, Feb. 1972.

[6] G. I. Vasilescu and T. Redon, "A new approach to sensitivity computation of microwave circuits," in Proc. IEEE Int. Circuits Syst. Symp., Helsinki, Finland, Jun. 1988, pp. 1167-1170.

[7] T. Redon and G. I. Vasilescu, "Second- and third-order sensitivities of microwave circuits," Electron. Lett., vol. 25, no. 9, pp. 607-609, Apr. 1989.

[8] V. A. Monaco and P. Tiberio, "Computer-aided analysis of microwave circuits," IEEE Trans. Microw. Theory Tech., vol. MTT-22, no. 3, pp. 249-263, Mar. 1974.

[9] O. Wing and J. V. Behar, "Circuit design by minimization using the Hessian matrix," IEEE Trans. Circuits Syst., vol. CAS-21, no. 5, pp. 643-649, Sep. 1974.
[10] F. Alessandri, M. Mongiardo, and R. Sorrentino, "New efficient full wave optimization of microwave circuits by the adjoint network method," IEEE Microw. Guided Wave Lett., vol. 3, no. 11, pp. 414-416, Nov. 1993.

[11] M. Mongiardo and R. Ravanelli, "Automated design of corrugated feeds by the adjoint network method," IEEE Trans. Microw. Theory Tech., vol. 45, no. 5, pp. 787-793, May 1997.

[12] J. Dobrowolski, Computer-Aided Analysis, Modeling, and Design of Microwave Networks. The Wave Approach. Norwood, MA: Artech House, 1996

[13] K. C. Gupta, R. Garg, and R. Chadha, Computer Aided Design of Microwave Circuits. Norwood, MA: Artech House, 1981.

[14] M. A. El Sabbagh and M. H. Bakr, "Analytical dielectric constant sensitivity of ridge waveguide filters," J. Electromagn. Waves Applicat., vol. 20, no. 3, pp. 363-374, 2006.

[15] M. Abramowitz and I. A. Stegun, Handbook of Mathematical Functions. New York: Dover, 1972, p. 884.

[16] R. Fletcher, Practical Methods of Optimization. New York: Wiley, 1980, vol. 1, Unconstrained Optimization.

[17] P. E. Gill, W. Murray, and M. H. Wright, Practical Optimization. London, U.K.: Academic, 1981.

[18] J. Nocedal and S. J. Wright, Numerical Optimization. New York: Springer-Verlag, 1999.

[19] Y. Rong, "Modeling of combline coaxial, ridge waveguide filters and multiplexers," Ph.D. dissertation, Dept. Elect. Comput. Eng., Univ. Maryland at College Park, College Park, MD, 1999.

[20] J. W. Bandler, "Optimization methods for computer-aided design," IEEE Trans. Microw. Theory Tech., vol. MTT-17, no. 8, pp. 533-552, Aug. 1969.

[21] J. W. Bandler and C. Charalambous, "Practical least $p$ th optimization of networks," IEEE Trans. Microw. Theory Tech., vol. MTT-20, no. 12, pp. 834-840, Dec. 1972.

[22] R. H. Byrd, P. Lu, and J. Nocedal, "A limited memory algorithm for bound constrained optimization," SIAM J. Sci. Stat. Comput., vol. 16, no. 5, pp. 1190-1208, 1995.

[23] C. Zhu, R. H. Byrd, and J. Nocedal, "L-BFGS-B: Algorithm 778: L-BFGS-B, FORTRAN routines for large scale bound constrained optimization," ACM Trans. Math. Softw., vol. 23, no. 4, pp. 550-560, Dec. 1997.

[24] M. A. El Sabbagh, H. T. Hsu, and K. A. Zaki, "Stripline transition to ridge waveguide bandpass filters," Progr. Electromagn. Res., vol. 40, pp. 29-53, 2003.

[25] HFSS. ver. 9.2.1, Ansoft, Pittsburgh, PA, 2004.

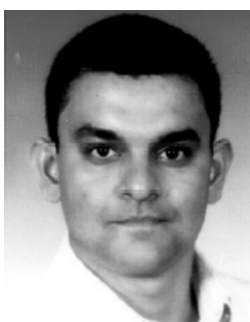

Mahmoud A. El Sabbagh (S'93-M'02) received the B.S. (with honors) and M.S. degrees in electrical engineering from Ain Shams University, Cairo, Egypt, in 1994 and 1997, respectively, and the Ph.D. degree from the University of Maryland at College Park, in 2002.

From 1994 to 1998, he was a Lecturer and Research Assistant with the Department of Electrical Engineering, Ain Shams University, where his research dealt with applications of superconductors in microwave circuits. In June 1998, he joined the Microwave Group, University of Maryland at College Park. From May 2001 to June 2002, he was a Guest Researcher with the National Institute of Standards and Technology (NIST) Gaithersburg, MD. From September 2002 to July 2003, he was a Visiting Scientist with the United Stated Department of Agriculture-Agricultural Research Service (USDA-ARS). Since October 2003, he has been an Assistant Professor with the Faculty of Engineering, Electronics and Communications Department, Ain Shams University. From December 2003 to January 2005, he led the microwave team of the National Authority for Remote Sensing and Space Sciences, Cairo, Egypt. In 2005, he was a Post-Doctoral Fellow with the Electrical and Computer Engineering Department, McMaster University. He is currently a Post-Doctoral Fellow with the Electrical and Computer Engineering Department, University of Waterloo, Waterloo, ON, Canada. His research interests include computer-aided design of microwave devices, microwave filters modeling and design, dielectric characterization, microwave remote sensing, and EM theory. 


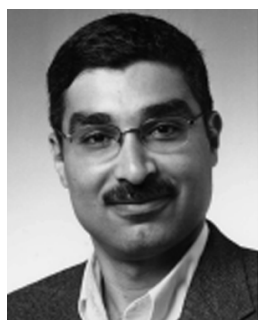

Mohamed H. Bakr (S'98-M'00) received the B.Sc. degree in electronics and communications engineering [with distinction (honors)] and M.Sc. degree in engineering mathematics from Cairo University, Cairo, Egypt, in 1992 and 1996, respectively, and the Ph.D. degree from McMaster University, Hamilton, ON, Canada, in 2000.

In 1997, he was a Student Intern with Optimization Systems Associates Inc. (OSA), Dundas, ON, Canada. From 1998 to 2000, he was a Research Assistant with the Simulation Optimization Systems (SOS) Research Laboratory, McMaster University. In November 2000, he joined the Computational Electromagnetics Research Laboratory (CERL), University of Victoria, Victoria, BC, Canada, as a Natural Sciences and Engineering Research Council of Canada (NSERC) Post-Doctoral Fellow. He is currently an Assistant Professor with the Department of Electrical and Computer Engineering, McMaster University. His research areas of interest include optimization methods, computer-aided design and modeling of microwave circuits, neural-network applications, smart analysis of microwave circuits, and efficient optimization using time-/frequency-domain methods.

Dr. Bakr was a recipient of the Premier's Research Excellence Award (PREA) presented by the Province of Ontario, Canada, in 2003.

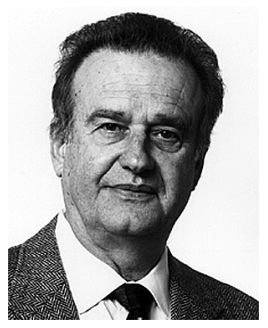

John W. Bandler (S'66-M'66-SM'74-F'78) was born in Jerusalem, on November 9, 1941. He received the B.Sc.(Eng.), Ph.D., and D.Sc.(Eng.) degrees from the University of London, London, U.K., in 1963, 1967, and 1976, respectively.

He joined Mullard Research Laboratories, Redhill, Surrey, U.K., in 1966. From 1967 to 1969, he was a Postdoctorate Fellow and Sessional Lecturer at the University of Manitoba, Winnipeg, Canada. He joined McMaster University, Hamilton, ON, Canada, in 1969. He was Chairman of the Department of Electrical Engineering and Dean of the Faculty of Engineering. He is currently Professor Emeritus in Electrical and Computer Engineering, directing research in the Simulation Optimization Systems Research Laboratory. He has authored or coauthored over 385 papers. He was a member of the Micronet Network of Centres of Excellence. He was President of Optimization Systems Associates Inc. (OSA), which he founded in 1983, until November 20, 1997, the date of acquisition of OSA by the Hewlett-Packard Company. OSA implemented a first-generation yield-driven microwave computer-aided design (CAD) capability for Raytheon in 1985, followed by further innovations in linear and nonlinear microwave CAD technology for the Raytheon/Texas Instruments Joint Venture MIMIC Program. OSA introduced the CAE systems RoMPE in 1988, HarPE in 1989, OSA90 and OSA90/hope in 1991, Empipe in 1992, and Empipe3D and EmpipeExpress in 1996. OSA created the product empath in 1996 which was marketed by Sonnet Software Inc. He is President of Bandler Corporation, which he founded in 1997. He joined the Editorial Boards of the International Journal of Numerical Modelling in 1987, the International Journal of Microwave and Millimeterwave Computer-Aided Engineering in 1989, and Optimization and Engineering in 1998. He was a Guest Editor of the International Journal of Microwave and Millimeter-Wave Computer-Aided Engineering Special Issue on "Optimization-Oriented Microwave CAD" (1997) He was Guest Coeditor of the Optimization and Engineering Special Issue on "Surrogate Modelling and Space Mapping for Engineering Optimization" (2001).

Dr. Bandler is a Fellow of the Canadian Academy of Engineering, the Royal Society of Canada, the Institution of Electrical Engineers, and the Engineering Institute of Canada. He is a member of the Association of Professional Engineers of the Province of Ontario, Canada, and the MIT Electromagnetics Academy. He was an associate editor of the IEEE TRANSACTIONS ON MiCROWAVE THEORY AND TECHNIQUES (1969-1974) and has continued serving as a member of the Editorial Board. He was guest editor of the IEEE TRANSACTIONS ON MicrowaVe THEORY AND TEChNiQues Special Issue on "Computer-Oriented Microwave Practices" (1974) and on "Automated Circuit Design Using Electromagnetic Simulators" (1997) and guest coeditor of the Special Issue on "Process-Oriented Microwave CAD and Modeling" (1992) and on "Electromagnetics-Based Optimization of Microwave Components and Circuits" (2004). He was chair of the MTT-1 Technical Committee on Computer-Aided Design. He was the recipient of the 1994 Automatic Radio Frequency Techniques Group Automated Measurements Career Award and the 2004 IEEE Microwave Theory and Techniques Society (MTT-S) Microwave Application Award. 